\title{
Un précurseur ? Christophe-Michel Ruffelet (11 janvier 1725-21 août 1806), chanoine et historien à l'époque des Lumières
}

\section{Olivier Charles}

\section{(2) OpenEdition \\ Journals}

\section{Édition électronique}

URL : http://journals.openedition.org/abpo/1285

DOI : $10.4000 /$ abpo. 1285

ISBN : 978-2-7535-1494-2

ISSN : 2108-6443

\section{Éditeur}

Presses universitaires de Rennes

\section{Édition imprimée}

Date de publication : 20 juillet 2004

Pagination : 89-118

ISBN : 978-2-7535-0031-0

ISSN : 0399-0826

\section{Référence électronique}

Olivier Charles, « Un précurseur? Christophe-Michel Ruffelet (11 janvier 1725-21 août 1806), chanoine et historien à l'époque des Lumières », Annales de Bretagne et des Pays de l'Ouest [En ligne],

111-2 | 2004, mis en ligne le 20 juillet 2006, consulté le 01 mai 2019. URL : http://

journals.openedition.org/abpo/1285; DOI : 10.4000/abpo.1285 


\title{
Un précurseur? Christophe-Michel Ruffelet (11 janvier 1725-21 août 1806), chanoine et historien à l'époque des Lumières
}

\author{
Olivier CHARLES \\ chercheur associé au CRHISCO \\ Université Rennes 2 - Haute-Bretagne
}

Les chapitres des cathédrales bretonnes du XIX ${ }^{\mathrm{e}}$ siècle et de la première moitié du XX $\mathrm{X}^{\mathrm{e}}$ siècle ont tous livré de belles figures de chanoines érudits. Les travaux des chanoines Mahé ou Le Mené à Vannes, Durville ou Bachelier à Nantes, Guillotin de Corson à Rennes, Abgrall ou Pérennes à Quimper - entre autres - restent de précieuses contributions à l'histoire ecclésiastique de la Bretagne ${ }^{1}$. La Révolution puis les lois de séparation, l'inquiétude suscitée par la confiscation ou la disparition d'objets et d'édifices ont ici joué un rôle déterminant en incitant les évêques à encourager les vocations d'historiens et d'archéologues : il y avait, pour eux, une certaine urgence à écrire l'histoire des paroisses, des évêchés ou des institutions religieuses, à étudier l'archéologie, afin de mieux connaître et tenter de mieux protéger un monde en train de disparaître. Les études des chanoines érudits s'inscrivent donc dans un large mouvement de publication de monographies historiques. Cette tradition peut ainsi être considérée comme l'expression d'une nouvelle sensibilité capitulaire qui aurait fait

1. GuIOMAR, Jean-Yves, Le Bretonisme. Les historiens bretons au XIXe siècles, Mayenne, Société d'Histoire et d'Archéologie de Bretagne, 1987; DANIEL, Tanguy, « Les chanoines de la Société Archéologique du Finistère ", Chrétiens de Basse-Bretagne et d'ailleurs. Les archives au risque de l'histoire. Mélanges offerts au chanoine Jean-Louis Le Floc'h, Société Archéologique du Finistère, Quimper, 1998, p. 491-496; GouRHAND, Jean, « L'abbé Joseph Mahé, premier érudit morbihannais " et LAUNAY, Marcel, « Prêtres érudits ou prêtres historiens? L'exemple nantais : XIX ${ }^{\mathrm{e}}-\mathrm{XX}{ }^{\mathrm{e}}$ siècles " dans TONNERRE, Noël-Yves (dir.), Chroniqueurs et historiens de la Bretagne du Moyen Âge au milieu du XXe siècle, Rennes, Presses Universitaires de Rennes, 2001, p. 125-142 et p. 223-232; HALLEREAU, Isabelle, " Le rôle du clergé dans l'émancipation de la science archéologique en Bretagne au XIX siècle ", Mémoires de la Société d'Histoire et d'Archéologie de Bretagne, t. LXXXI, 2003, p. 371-401. 
défaut à leurs prédécesseurs. Les chapitres bretons d'Ancien Régime ne sont en effet guère réputés pour avoir été des viviers d'intellectuels d'envergure, à la différence de leurs homologues de Guyenne ou du Cambrésis ${ }^{2}$. Pourtant, il semble possible d'y rencontrer l'archétype de cette figure du chanoine-érudit.

Aujourd'hui encore, les Archives départementales des Côtes-d'Armor comme le séminaire de Saint-Brieuc conservent des ouvrages curieusement marqués d'une étiquette bleue frappée d'un " $\mathrm{R}$ ». Il s'agit d'un vestige du legs que fit Christophe-Michel Ruffelet de sa bibliothèque à l'évêque de Saint-Brieuc pour doter le séminaire du diocèse ${ }^{3}$. Mais, pour certains, près de deux siècles après sa disparition, plus que le propriétaire d'une imposante bibliothèque, Ruffelet est avant tout l'auteur des Annales briochines ${ }^{4}$ qui rendent toujours de précieux services à l'historien du début du $\mathrm{XXI}^{\mathrm{e}}$ siècle... si bien que l'on oublie qu'il fit partie de la vingtaine de chanoines de la cathédrale de Saint-Brieuc ${ }^{5}$ à la veille de la Révolution.

Il est vrai que les chanoines d'Ancien Régime sont méconnus. Cruellement brocardés par les auteurs du temps qui ne voient en eux que d'inutiles oisifs s'épuisant dans de sombres querelles et discussions sans fin $^{6}$, antithèses du bon prêtre décrit par les philosophes ${ }^{7}$, ils sont définitivement condamnés par les Constituants qui suppriment les chapitres cathédraux et collégiaux en 1790. Souvent assimilés à leurs confrères du XIX ${ }^{\mathrm{e}}$ siècle, on leur accole volontiers une image de prêtres méritants achevant dans la léthargie, au sein "d'une maison de retraite vouée à la prière publique $^{8}$ ", une existence entièrement consacrée à Dieu. Nécessairement âgés, ils vivotent et, chantonnant dans leur stalle, les mains sur le bedon, ils pensent avant tout au dîner qui les attend ${ }^{9}$. D'abord contesté à

2. Pour les premiers, LouPÈs, Philippe, Chapitres et chanoines de Guyenne aux XVII et XVIII siècles, Paris, Éditions de l'EHESS, 1985, p. 318-319; pour les seconds, données communiquées par Gilles Deregnaucourt.

3. Arch. dép. des Côtes-d'Armor, 3 E 3, 126, Étude Conan, testament de C.-M. Ruffelet, 30 juillet 1806.

4. RUFFELET, Christophe-Michel, Annales briochines, ou abrégé chronologique de l'histoire ecclésiastique, civile et littéraire du diocèse de Saint-Brieuc, enrichi de plusieurs notes historiques, géographiques et critiques, Saint-Brieuc, Mahé, 1771. Cette édition est un tout petit volume - in $24^{\circ}$ - aux caractères microscopiques.

5. En qualité de clerc attaché au service de la première église du diocèse il était titulaire d'un bénéfice appelé canonicat dont la rétribution est la prébende. Le chapitre cathédral de Saint-Brieuc abrite vingt prébendes, dont quatre sont attribuées respectivement à l'évêque, au principal du collège, à la psallette, au duc de Penthièvre.

6. Voir par exemple Le lutrin de Nicolas Boileau ou Le conseil tenu par les rats de Jean de La Fontaine.

7. SAIGE, P., Le "bon prêtre » dans la littérature française d'Amadis de Gaule au Génie du christianisme, Genève, Droz, 1951, p. 263-273; ou pour un exemple précis, RESTIF de LA Bretonne, Nicolas, La vie de mon père, Paris, Taillandier, 1929, p. 204-205.

8. PIERRARD, Pierre, La vie quotidienne du prêtre français au $\mathrm{XIX}^{e}$ siècle, 1801-1905, Paris, Hachette, 1986, p. 135.

9. Romains, Jules, Les Hommes de bonne volonté. T. 3, Les amours enfantines, réédition, Paris, Robert Laffont-Bouquins, 1988, t. 1, p. 316. 
Tréguier ${ }^{10}$, ce portrait doit être sinon effacé du moins sérieusement nuancé à l'échelle de la Bretagne ${ }^{11}$. Pour ces hommes en général dans la force de l'âge, la prébende n'est en effet pas la sinécure que l'on croit; par ailleurs, loin de se désintéresser du monde qui les entoure, nombre d'entre eux en suivent les débats, s'attachent à le mieux connaître et en adoptent les habitudes de consommation.

C'est à ce double titre - d'érudit et de chanoine - et à la lumière d'un monde capitulaire breton désormais mieux connu que le dossier de Christophe-Michel Ruffelet mérite d'être réouvert ${ }^{12}$. Le caractère insaisissable du personnage semble nous protéger d'une quelconque illusion biographique $^{13}$ : écrits en partie disparus, correspondance introuvable, absence de traces dans les archives familiales, non enregistrement de la prise de possession de l'un de ses canonicats dans les registres d'insinuations ecclésiastiques, indigence des archives du premier chapitre auquel il a appartenu... Finalement, le laconisme de la plaque apposée à l'entrée d'une petite rue briochine illustre bien ce paradoxe : Christophe-Michel Ruffelet est un illustre inconnu. Par conséquent, plus qu'une biographie au sens classique, cette étude, en superposant trois portraits complémentaires du personnage - un intellectuel, un historien, un chanoine -, se veut une réflexion sur les relations entretenues par un homme avec le milieu dont il est issu, avec la société dans laquelle il vit et, plus largement, sur les relations entre l'institution capitulaire et les élites locales. Chacun de ces éclairages, fondé sur des sources aux statuts différents, permet en effet de sortir de l'ombre un chanoine à l'inlassable curiosité ainsi que son univers social et culturel, certainement déterminant pour comprendre ses ambitions d'historien dans un monde en mutation.

\section{L'intellectuel}

Cet aspect de la personnalité de Christophe-Michel Ruffelet apparaît au travers des nombreux ouvrages qu'il a, comme nombre de ses homologues,

10. MinoIs, Georges, Un échec de la Réforme catholique en Basse-Bretagne : le Trégor du $X V I^{e}$ au XVIII siècle, thèse dactylographiée, Université Rennes 2 - Haute-Bretagne, 1984, t. IV, p. 811; MinoIs, Georges, "Clercs et inventions techniques d'après les mémoires de l'Académie Royale des Sciences de Paris, 1666-1770 ", Annales de Bretagne et des Pays de l'Ouest, t. 94, 1987, p. 431-449.

11. Charles, Olivier, Chanoines de Bretagne. Carrières et cultures d'une élite cléricale au siècle des Lumières, Rennes, Presses Universitaires de Rennes, 2004, 456 p. Sauf précision contraire, les données concernant le monde capitulaire breton proviennent de cet ouvrage.

12. La seule étude le concernant date de 1866 et est avant tout une publication d'extraits de lettres et de travaux historiques. Du BoIS DE LA VILLERABEL, Arthur, "Le chroniqueur Ruffelet ", Mémoires de la Société Archéologique des Côtes-du-Nord, t. II, 1866, p. 129198, 255-282 et 383-423. La notice dans LEVoT, Pierre, Biographie bretonne. Recueil de tous les Bretons qui se sont fait un nom, Vannes, Cauderan, t. II, 1857, p. 794-795 ne fournit pas d'information supplémentaire.

13. BouRdiEu, Pierre, "L'illusion biographique ", Actes de la Recherche en Sciences Sociales, ${ }^{\circ}$ 62-63, janvier 1986, p. 69-72. 
progressivement accumulés. Beaucoup de chanoines bretons compensent en effet l'absence de véritable bibliothèque capitulaire par l'acquisition de livres ${ }^{14}$. Des recherches menées pour les chapitres de Nantes, Rennes et Saint-Pol-de-Léon montrent que plus de $80 \%$ des chanoines possèdent des bibliothèques au XVIII ${ }^{\mathrm{e}}$ siècle. Ruffelet est cependant un cas à part puisqu'il dispose - avec plus de 4000 volumes - de la plus grande bibliothèque connue de chanoines en Bretagne ${ }^{15}$. Elle surclasse en effet celles du Léonard Jean-François Le Grand de Tromelin - 1714 volumes -, du Trégorrois Jacques-Louis Guino - 1454 volumes -, des Nantais Jean Barrin de La Galissonière - 1371 volumes - et Jérôme Le Febvre de Laubrière - 1300 volumes -, du Rennais Germain Bossard de La Rossignolière - 1312 volumes ${ }^{16}$. Par ailleurs, aucune bibliothèque particulière des villes de l'Ouest ${ }^{17}$, à l'exception notable de celle qui est saisie au début de la

14. Les ouvrages communs - peu nombreux - se trouvent dans les archives : le terme de bibliothèque paraît donc excessif. À Rennes, les deux volumes de l'Histoire de Bretagne, donnés par les États y sont rangés, Arch. dép. d'Ille et Vilaine, G 202 C, délibération du 23 décembre 1707. À Nantes, les commissaires chargés de dresser l'état des revenus et des dépenses du chapitre indiquent que les chanoines «n'ont point de bibliothèque commune, si on excepte les registres de leurs délibérations et environ une centaine de volumes de différents formats concernant l'histoire de la province, la jurisprudence et la discipline ecclésiastique ", ce qui est peu, Arch. dép. de Loire-Atlantique, Q 33, déclaration des revenus du chapitre cathédral de Nantes, 1790. À Saint-Malo, vingt et un volumes (dix titres), dont la moitié concernent l'histoire de la Bretagne, sont conservés dans les archives, Arch. dép. d'Ille-et-Vilaine, $1 \mathrm{Q}$ 663, procès verbal de dépôt à la bibliothèque de livres provenant du chapitre, 9 messidor an III. À Saint-Pol-de-Léon, la situation est moins claire. En effet, en 1747, le chapitre dépêche des députés pour assister à l'inventaire des biens d'un chanoine décédé et récupérer quelques livres " comme étant de la bibliothèque du chapitre ", Arch. dép. du Finistère, 23 B 358, inventaire des biens de Launay, 14 avril 1747.

15. L'inventaire de l'an II l'estimait à 4112 volumes (Arch. dép. des Côtes d'Armor, $1 \mathrm{Q}$ 517, inventaire des biens de Ruffelet, 12 messidor II); celui de 1810 à 4817 (Arch. dép. des Côtes d'Armor, 1 Q 517, catalogue des livres légués à l'évêché de Saint-Brieuc pour le séminaire par feu M. l'abbé Ruffelet, chanoine, 18 janvier 1810). Tous ces livres sont rentrés dans la bibliothèque du séminaire lorsqu'elle a été constituée par Le Groing de La Romagère en 1813, " une étiquette de papier bleuâtre collée à l'intérieur [des volumes] et présentant dans un encadrement la lettre $\mathrm{R}$, initiale du donateur " permettant de les reconnaître (Arch. dép. des Côtes-d'Armor, V 800, procès-verbal d'expertise de la bibliothèque Ruffelet, 15 novembre 1883). Un nouveau comptage en 1883 fait état de seulement 3459 volumes car " à la suite de dons faits à la bibliothèque du grand séminaire, la plupart des ouvrages qui manquent à la collection Ruffelet, et qu'on possédait en double, ont servi à enrichir et à compléter les bibliothèques des écoles secondaires ecclésiastiques " (Arch. dép. des Côtes-d'Armor, V-800, procès-verbal d'expertise de la bibliothèque Ruffelet, 15 novembre 1883). En 1912, après un nouvel inventaire de la bibliothèque du séminaire lié aux procédures d'application de la loi de séparation, un certain nombre d'ouvrages entrent dans la bibliothèque des Archives départementales (Arch. dép. des Côtes-d'Armor, - 3 T 13, loi de séparation de 1905, inventaires des bibliothèques et mobiliers ecclésiastiques).

16. Arch. dép. du Finistère, 23 B 369, DANIEL, René, " La bibliothèque de Jacques-Louis Guino ", Bulletin de la Société Archéologique du Finistère, t. 98, 1972, p. 265-296, Arch. dép. de Loire-Atlantique, B 9972 et 6907, Arch. dép. d'Ille-et-Vilaine, 4 B 4817.

17. QuÉNIART, Jean, Culture et société urbaines dans la France de l'Ouest au XVIII siècle, Paris, Klincksieck, 1978, p. 182-307. 
Révolution chez les Robien ${ }^{18}$, ne semble rivaliser avec elle. Au sein des élites urbaines bretonnes, Ruffelet est donc bien l'un des plus gros propriétaires de livres de son temps.

La source utilisée ici pour étudier sa bibliothèque soulève cependant un problème important. En effet, tardif, l'inventaire de 1810 pourrait ne refléter qu'imparfaitement la bibliothèque de Ruffelet. En fait, ses auteurs ont scrupuleusement référencé ses ouvrages marqués de la fameuse étiquette bleue frappée du « $\mathrm{R}$ ». Par ailleurs, les livres inventoriés dans l'inventaire de 1794 figurent dans celui de 1810. L'intérêt est donc de pouvoir étudier une bibliothèque complétée par son propriétaire jusqu'à sa mort par delà la Révolution, après qu'elle lui aura été rendue par les autorités. Plus classiquement, l'inventaire Ruffelet est également justiciable des critiques opposées à une source qui ne restitue qu'imparfaitement les centres d'intérêt et le profil intellectuel d'un individu. Outre le fait qu'un livre possédé n'est pas forcément un livre lu, qu'il est difficile de déterminer s'il est une acquisition ou un héritage, il ne faut pas oublier que toute bibliothèque peut comporter des couches fossiles révélatrices d'un savoir dépassé que son propriétaire ne consulte plus. À cela, il faudrait ajouter les limites liées à la rédaction même du document : erreurs de comptage des tomes, non identification d'auteurs... Photographie à un moment donné du contenu de la bibliothèque, l'inventaire ne rend malheureusement pas exactement compte de l'histoire de sa constitution ni de l'évolution des centres d'intérêt de son propriétaire ${ }^{19}$. L'examen des dates d'édition des ouvrages permet néanmoins de lever quelques réserves.

$90 \%$ des titres ont été publiés au cours du XviII ${ }^{\mathrm{e}}$ siècle, dont plus des deux tiers après 1750, année des 25 ans de Christophe-Michel. Cet aspect contemporain de la bibliothèque doit être rapproché de ce que disent de lui ses biographes. En effet, selon eux, il ne rechigne pas à acquérir des livres : lorsque sa nièce lui demandait la permission de lire un de ses ouvrages, " il ouvrait tranquillement sa bourse et en tirait le prix du volume en disant "M. Prud'homme vend ce livre-là, et vous savez le chemin de sa maison. Un livre se donne, mais ne se prête pas" ${ }^{20}$ ". Il semble donc plausible que tout au long de son existence, et " dès ses plus jeunes années ", Ruffelet ait acheté lui-même une très grande partie de ses livres jusqu'à " former, sur toutes les branches des connaissances humaines, une très belle bibliothèque ${ }^{21}$ ". Cette dernière dévoile au passage un autre aspect de la personnalité de son propriétaire. S'il y a incontestablement une dimen-

18. AuBERT, Gauthier, Le Président de Robien. Gentilhomme et savant dans la Bretagne des Lumières, Rennes, Presses Universitaires de Rennes, 2001, p. 309. La bibliothèque Robien contient 4308 volumes; celle de Ruffelet, 4112 lorsqu'elle est saisie en 1794.

19. Sur ces problèmes aujourd'hui classiques, on peut consulter QuÉNIART, Jean, Culture et société urbaines..., op. cit., p. 159-163 ou CHARTIER, Roger, Lecteurs et lectures dans la France d'Ancien Régime, Paris, Seuil, 1987, p. 166-167.

20. RuFfElEt, Christophe-Michel, Annales briochines, publiées par Sigismond Roparz, Saint-Brieuc, Prud'homme, 1851, p. XIII.

21. LeVot, Pierre, Biographie bretonne..., op. cit., t. II, p. 795. 
Figure 1 - Dates d'édition des ouvrages de C.-M. Ruffelet

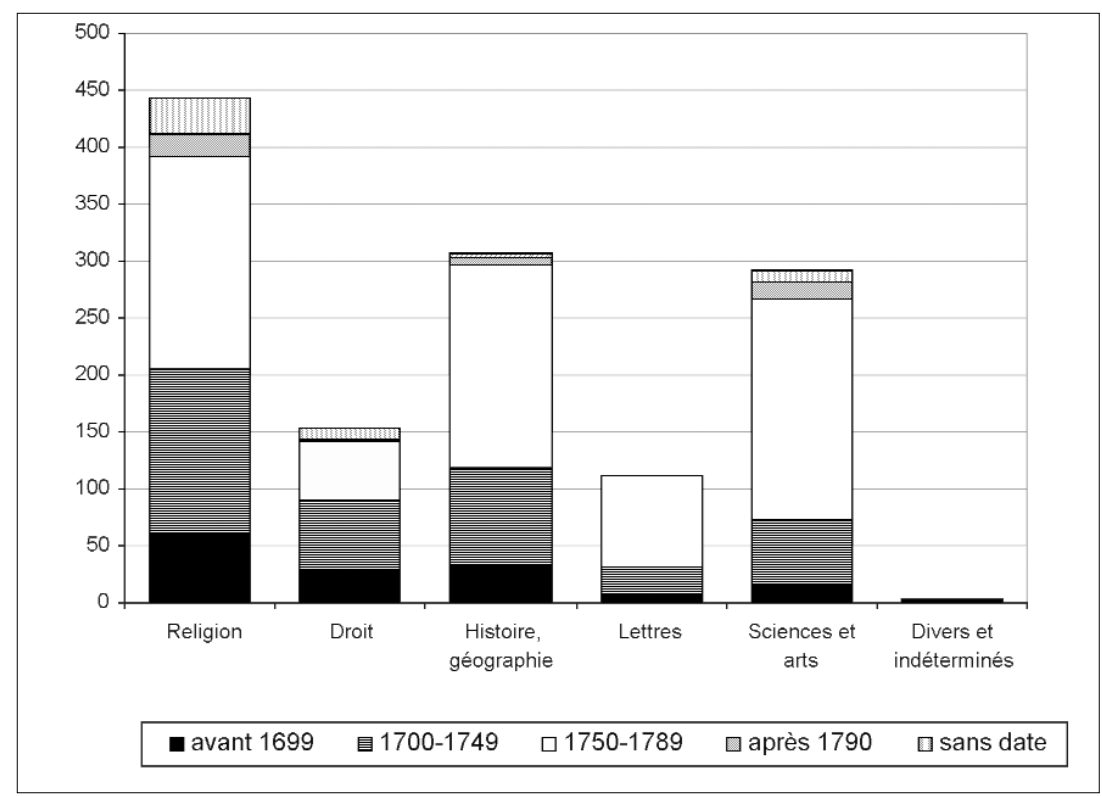

sion de collectionneur maniaque chez lui, son rapport aux livres est aussi empreint d'une réelle vénération de la connaissance et est certainement sous-tendu par la croyance en la possibilité de rassembler dans une même pièce tous les fruits de la réflexion humaine. Ses efforts pour éviter la dispersion des livres doivent être interprétés en ce sens : offrir un livre au lieu de le prêter, demander la restitution de la bibliothèque aux autorités révolutionnaires ${ }^{22}$, exiger par testament que l'évêque auquel il la lègue ne la disperse pas ${ }^{23}$. Ces constats formulés, il est possible de présenter les grandes caractéristiques de cette immense bibliothèque.

22. Après son incarcération Ruffelet demande à rentrer en possession de sa bibliothèque destinée à rejoindre la future bibliothèque nationale : "Voudriez-vous bien citoyens me permettre d'y rentrer. La permission que vous donneriez à cet égard ne pourrait être que très utile et avantageuse à la bibliothèque. Vous n'ignorez pas tous les dégâts que les rats, les souris, les autres insectes, le défaut même d'air peuvent y causer. Elle a absolument besoin de quelqu'un qui en prenne soin. Je puis vous assurer citoyens que vous ne pouvez lui trouver un meilleur gardien que moi, plus attentif, plus vigilant et en même temps plus reconnaissant ". Le Directoire du district de Saint-Brieuc " considérant que cette collection de livres est précieuse et ne peut être conservée avec plus de soin que par celui qui l'a formée " lui en confie la garde car " il ne peut y avoir aucun danger à confier la garde de cette bibliothèque à son ancien propriétaire ", Arch. dép. des Côtesd'Armor, 1 Q 517, lettre de Ruffelet à l'administration municipale de Port-Brieuc.

23. Léguée à Jean-Baptiste Cafarelli et à ses successeurs en 1806, la bibliothèque doit théoriquement être enfermée dans " un ou plusieurs appartements propres à cet effet, dont les clefs seront remises aux susdits évêques de Saint-Brieuc, sans qu'aucun des dits livres puissent en être distrait, prêté, ni sortir hors de ladite bibliothèque ", Arch. dép. des Côtes d'Armor, 3 E 3, 126, étude Conan, testament de C.-M. Ruffelet, 30 juillet 1806. 
Figure 2 - Composition de la bibliothèque

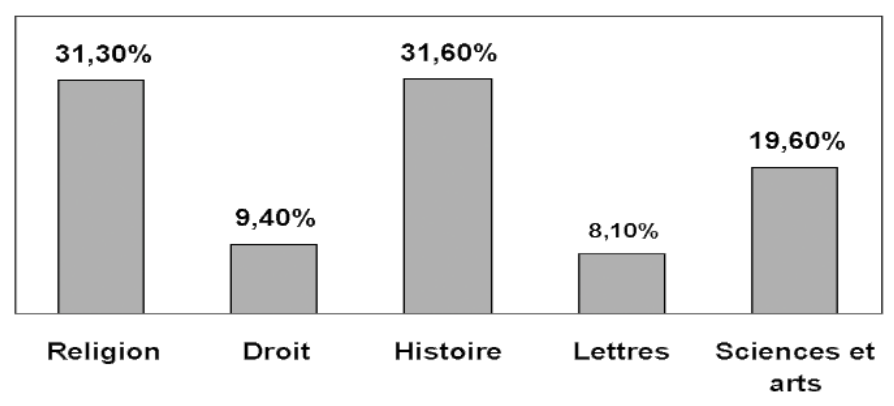

Les rubriques religion ${ }^{24}$ et histoire regroupent plus de $60 \%$ des volumes et $57 \%$ des titres. Le rayon religieux est dominé par les ouvrages de controverse, de spiritualité et de pastorale alors que, comme chez beaucoup de prêtres de la fin de l'Ancien Régime, les pères et la littérature conciliaire occupent, sans être ignorés, une place très restreinte. Ce rayon révèle des compétences que l'évêque de Saint-Brieuc Régnauld de Bellescize met à profit en demandant à Ruffelet de rédiger le Propre du diocèse en $1782^{25}$. De même ampleur, le secteur historique fort de ses 1511 volumes (308 titres) est le plus étoffé au sein du monde des chanoines bretons. La seconde bibliothèque d'histoire, celle du Nantais Barrin, ne contient en effet que 405 volumes (200 titres) en $1719^{26}$. Particulièrement intéressé par l'histoire moderne (602 volumes pour 133 titres), Ruffelet ne néglige ni l'histoire ancienne ni l'histoire ecclésiastique. Loin derrière - et pourtant particulièrement importante lorsqu'on la compare avec celle de ses confrères -, l'hétérogène catégorie des sciences et arts est dominée par la philosophie, la physique, les sciences naturelles et les mathématiques. L'analyse montre que son propriétaire ne méconnaît par ailleurs pas les débats politiques, économiques, agricoles et éducatifs.

En réalité, plusieurs chanoines possèdent des bibliothèques de ce type au sein desquelles le secteur religieux tout en restant important est concurrencé par le progrès d'autres domaines de la connaissance. Comme chez nombre de ses confrères, il est possible de relativiser l'affaiblissement de la sphère religieuse chez Ruffelet : le rayon religieux complété par les ouvrages de droit canon, de droit ecclésiastique et d'histoire ecclésiastique comprend en effet plus de $40 \%$ des volumes et $57 \%$ des titres.

24. Qui reste la rubrique dominante chez les chanoines bretons d'Ancien Régime.

25. DUBOIS DE LA VILLERABEL, Arthur, « Le chroniqueur Ruffelet », art. cit., p. 150. L'analyse du Propre serait peut-être de nature à préciser les positions de Ruffelet en matière de liturgie et de théologie. C'est une piste, suggérée par Georges Provost, que nous n'avons pu exploiter pour l'instant.

26. Arch. dép. de Loire-Atlantique, B 9972. Le rayon historique représente 36 \% des volumes de sa bibliothèque de 1371 volumes. Barrin est chanoine de Nantes de 1685 (Arch. dép. de Loire-Atlantique, G 839, f $^{\circ} 19$ r $^{\circ}$ ) à 1718 (Arch. dép. de Loire-Atlantique, G $844, \mathrm{f}^{\circ} 296 \mathrm{r}^{\circ}$ ). 
Figure 3 - Composition de la bibliothèque après recomposition ${ }^{27}$

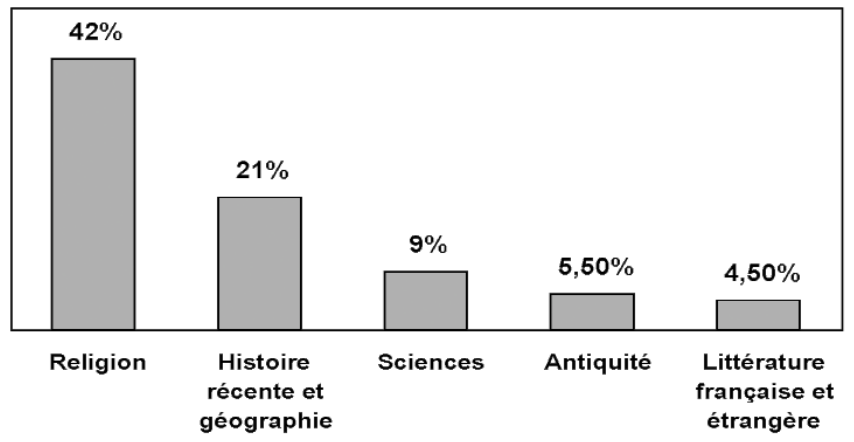

Par conséquent, la véritable originalité de Ruffelet réside dans l'adéquation de sa bibliothèque avec certaines évolutions récentes du savoir, que la possession de "feuilles supplétives de l'Encyclopédie " et de l'Encyclopédie par ordre de matières de Panckoucke ne saurait résumer. Le premier indice semble en être la place particulière occupée par les dictionnaires. 76 titres ( $6 \%$ de la bibliothèque) couvrent de nombreux domaines avec une prédilection pour l'histoire et la géographie, les sciences et les lettres. Ces multiples dictionnaires révèlent un schéma de pensée, une manière d'aborder et de s'approprier le savoir. Ce sont des outils pratiques qui permettent de vulgariser les connaissances, d'examiner librement une question en dehors de tout système. Les dictionnaires de langue, de grammaire, de synonymes, les nombreuses grammaires traduisent le besoin d'outils linguistiques, bien utiles à un écrivain.

Par ailleurs, à une époque où la préoccupation pour la science et sa transmission devient centrale chez les Encyclopédistes ${ }^{28}$, où le goût pour la science n'est pas rare dès le milieu du siècle au sein d'une élite bretonne cultivée ${ }^{29}$, Ruffelet est de ces chanoines attentifs à ses développements. Elle représente $9 \%$ des volumes et $13 \%$ des titres de sa bibliothèque quand chez nombre de chanoines, sans être absente, elle ne dépasse pas quelques dizaines de volumes ${ }^{30}$.

27. C'est en effet en procédant à des regroupements de rubriques appartenant à des catégories bibliographiques différentes que l'on peut approcher les catégories mentales et " décrypter les cultures qui se cachent derrière les livres " (QUÉNIART, Jean, Culture et société urbaines..., op. cit., p. 183). Il s'agit donc ici de faire apparaître les principaux domaines qui traduisent les évolutions de l'époque : tous les livres ne sont donc pas pris en compte. Le secteur religieux regroupe les ouvrages religieux, les livres de droit ecclésiastique, de droit canon et d'histoire ecclésiastique; le secteur ancien ceux de droit ancien civil, d'histoire ancienne, de lettres anciennes, de rhétorique, de poésie ancienne, de philosophie ancienne...

28. DHOMBRES, Jean (dir.), La Bretagne des savants et des ingénieurs. 1750-1825, Rennes, Ouest-France, p. 14.

29. AUBERT, Gauthier, Le président de Robien..., op. cit., p. 171.

30. 103 volumes chez Le Grand de Sparfel à Saint-Pol-de-Léon (Arch. dép. du Finistère, 23 B 369), 91 chez Bossard à Rennes (Arch. dép. du Finistère, 4 B 4817), 35 chez Gauvain à Nantes (Arch. dép. de Loire-Atlantique, B 9989) pour les mieux pourvus. 
Tableau 1 - Les dictionnaires de C.-M. Ruffelet

\begin{tabular}{|l|c|c|}
\hline & Volumes & Titres \\
\hline Histoire (généralités) & 124 & 20 \\
Histoire ecclésiastique & 6 & 3 \\
Histoire moderne & 2 & 1 \\
Mathématiques & 4 & 3 \\
Sciences naturelles & 30 & 4 \\
Physique-chimie & 19 & 6 \\
Médecine & 6 & 1 \\
Lettres & 11 de latin & 23 dont 7 de latin \\
Agriculture & 12 & 2 \\
Économie & 6 & 2 \\
Commerce & 5 & 2 \\
Peinture, dessin... & 2 & 2 \\
Éducation & 2 & 1 \\
Sciences et arts (généralités) & 29 & 3 \\
Divers & 15 & 76 \\
Total & 302 & \\
\hline
\end{tabular}

Tableau 2 - Les sciences dans la bibliothèque de C.-M. Ruffelet

\begin{tabular}{|l|c|c|}
\hline & Volumes & Titres \\
\hline Sciences naturelles & 173 & 41 \\
Physique - chimie & 116 & 49 \\
Mathématiques & 64 & 40 \\
Astronomie-météorologie & 40 & 21 \\
Médecine & 24 & 12 \\
Botanique & 7 & 4 \\
Total & 424 & 167 \\
\hline
\end{tabular}

Le moralisant Spectacle de la nature de l'abbé Pluche semble bien isolé face aux nombreux volumes de Buffon et Valmont de Bomare ${ }^{31}$ parmi les ouvrages généraux de sciences naturelles. Ruffelet possède par ailleurs un intéressant rayon consacré à la minéralogie et à la géologie où se côtoient les œuvres de l'abbé Haüy, de Dolomieu ${ }^{32}$ et de Valmont ainsi que le Voyage physique dans les Pyrénées. Autant de lectures qui permettent de situer notre chanoine parmi les modernes dans les débats sur les origines de la Terre et des êtres vivants. Très intéressé par les sciences physiques, il possède la Description et usage d'un cabinet de physique expérimentale et de

31. Ruffelet possède 56 volumes (avec figures) de l'Histoire naturelle de Buffon, qui ouvre une brèche dans les conceptions bibliques des origines de la Création. Le naturaliste Jacques Valmont de Bomare (1731-1807) a parcouru une bonne partie de l'Europe avant de donner des cours très suivis d'histoire naturelle. Il est l'auteur d'un Dictionnaire d'histoire naturelle qui connut plusieurs éditions (Ruffelet possède les éditions de 1778 et de 1791 et un abrégé du dictionnaire).

32. Collaborateur de Lavoisier, l'abbé René Just Haüy (1743-1822) est le créateur de la minéralogie moderne. Dieudonné de Gratet de Dolomieu (1750-1801) est minéralogiste et a donné son nom au massif des Dolomites. 
nombreux cours ou traités dus à Haüy, Nollet, Deslandes, Régnault, Sigaud, Polinière $^{33}$. Esprit encyclopédique, il s'intéresse aussi aux avancées les plus récentes de cette discipline : il connaît les travaux de l'abbé Bertholon dans le domaine de l'électricité - sujet qui connaît un franc succès à travers les milieux les plus cultivés d'Europe -, possède des ouvrages consacrés à l'étude de l'air, de l'eau et de la glace. Son esprit scientifique, qui le conduit à s'intéresser de près aux théories newtoniennes sans forcément renier Descartes (il le lit et possède le Traité de paix entre Descartes et Newton), est confirmé par son rayon astronomie où l'Histoire du ciel de Pluche qui critique et les idées de Descartes et celles de Newton semble n'être qu'une concession à la formation initiale peu audacieuse d'un ecclésiastique ${ }^{34}$, tellement elle paraît dépassée à côté des travaux de l'astronome Jérôme de Lalande ${ }^{35}$. Des ouvrages de gnomonique et d'horlogerie complètent ces derniers et la présence de l'Essai sur l'horlogerie de l'horloger suisse Ferdinand Berthoud est tout sauf anecdotique puisque l'auteur, qui considère que l'horlogerie est une science qui " n'est autre chose que l'application du temps à l'espace ", rédigera plusieurs articles dans l'Encyclopédie ${ }^{36}$. En mathématiques, les Cours, Traités et autres Éléments, dont certains de la plume de grands savants de l'époque - Bézout, Camus, Clairaut, Le Gendre ou le chanoine Mazéas ${ }^{37}$ - font presque oublier les ouvrages toujours pratiques de Barrême.

33. L'abbé Jean Antoine Nollet (1700-1770) démontra que le son était conduit par l'eau et proposa d'utiliser l'électricité à des fins thérapeutiques. Il pratiqua le premier un enseignement de physique expérimentale au collège de Navarre. André-François Boureau Deslandes (1690-1757) fut membre de l'Académie des sciences de Berlin. Scientifique cartésien, le jésuite Noël Régnault (1683-1762) contribua à la vulgarisation des sciences exactes - notamment de la physique - en France. Joseph-Aignan Sigaud de La Fond (17301810) entreprit des études de médecine avant que les cours de Nollet ne décident de sa réorientation vers la physique expérimentale. S'intéressant à l'électricité et aux gaz, il succéda à Nollet à la chaire de physique expérimentale à Louis le Grand. Le mathématicien et physicien Pierre Polinière (1671-1734) se rendit célèbre par ses travaux sur la lumière électrique et un cours entrecoupé d'expériences professé devant Louis XV.

34. L'abbé Pierre-Nicolas Bertholon (1742-1800) se passionne pour les inventions de Franklin, contribue à répandre le paratonnerre à Lyon puis à Paris et consacre ses travaux aux phénomènes électriques. L'abbé Noël-Antoine Pluche (1688-1761) défend " une théologie naturelle " et, " convaincu que la physique deviendra saine à mesure qu'elle se rapprochera de l'Écriture sainte ", il s'efforce de mettre la science au service de la foi.

35. Jérôme Lefrançois de Lalande (1732-1807) multiplie les relevés à partir de l'observatoire du collège de France puis de celui de l'École militaire de Paris. Il en rassemble les résultats dans l'Histoire céleste française qui indique les positions de 50000 étoiles.

36. Article « horlogerie » dans DeLon, Michel (dir.), Dictionnaire européen des Lumières, Paris, PUF, 1997, p. 556

37. Étienne Bézout (1730-1783) a notamment étudié les méthodes de résolution des équations du troisième et du quatrième degré. Charles Camus (1699-1768), collaborateur de Maupertuis et de Cassini, s'intéresse aux mathématiques et à la physique. Astronome et mathématicien, Alexis Claude Clairaut (1713-1765) développa son œuvre à partir des travaux de Newton. Adrien Marie Le Gendre (1752-1833) travaille sur les intégrales elliptiques et eulériennes, et approfondit l'étude de la trigonométrie rectiligne et sphérique. Guillaume Mazéas, chanoine de Vannes de 1759 (Arch. dép. du Morbihan, 42 G 17, $\mathrm{f}^{\circ} 246 \mathrm{v}^{\circ}$ ) à 1775 (Arch. dép. du Morbihan, 83 G 2), est plus connu pour son intérêt pour l'électricité ou la chimie. 
Expression d'une évolution générale, le déclin de l'antiquité est particulièrement accusé chez Ruffelet.

Tableau 3 - Tradition et modernité dans la bibliothèque de C.-M. Ruffelet

\begin{tabular}{|lr|r|}
\hline \multicolumn{2}{|l|}{ Littérature ancienne } & \multicolumn{2}{l|}{ Littérature française } \\
& 61 volumes/15 titres & 223 volumes/42 titres \\
\hline \multicolumn{2}{|l|}{ Philosophie ancienne } & Philosophie moderne et antiphilosophie \\
& 7 volumes/2 titres & 108 volumes/47 titres \\
\hline Droit ancien & 9 volumes/2 titres & $\begin{array}{r}\text { Droit civil moderne } \\
285 \text { volumes/84 titres }\end{array}$ \\
\hline Histoire ancienne & 171 volumes/42 titres & $\begin{array}{r}\text { Histoire de France et des pays étrangers } \\
980 \text { volumes/193 titres }\end{array}$ \\
\hline
\end{tabular}

La littérature ancienne pèse en effet bien peu face à la littérature française, où l'on remarque quatre volumes du chanoine malouin Nicolas Trublet ${ }^{38}$. La philosophie ancienne fait pâle figure à côté de la philosophie moderne où Descartes et Mallebranche côtoient Condillac. Quant au droit ancien, il est surclassé par le droit civil moderne dont la plus belle pièce est incontestablement L'esprit des lois de Montesquieu. Enfin, l'histoire ancienne ne peut rivaliser avec l'histoire de France et des pays étrangers.

Tableau 4 - L'ouverture sur le monde dans la bibliothèque de C.-M. Ruffelet

\begin{tabular}{|l|c|c|}
\hline & Volumes & Titres \\
\hline Histoire étrangère & 186 & 37 \\
Géographie & 191 & 31 \\
Total & $8 \%$ & $5 \%$ \\
\hline
\end{tabular}

Dernière manifestation de cette modernité intellectuelle, l'ouverture sur le monde est indéniable chez Ruffelet, et nettement plus poussée que chez les autres prébendés puisque seul le Nantais Gauvain ${ }^{39}$ le surclasse. Notons toutefois qu'il ne possède pas d'œuvres littéraires en langues étrangères et que le dictionnaire et la grammaire italienne de Veneroni peuvent n'être que des ouvrages utilitaires liés à un séjour romain, assez classique chez les ecclésiastiques. Au rang des auteurs étrangers ne figure, semble-t-il, que

38. Il est chanoine de la cathédrale de Nantes de 1741 à 1744 (Arch. dép. de LoireAtlantique, G 849, $\mathrm{f}^{\circ} 281 \mathrm{v}^{\circ}$ et $850, \mathrm{f}^{\circ} 429 \mathrm{r}^{\circ}$ ) puis de Saint-Malo de 1745 à 1770 (Arch. dép. d'Ille-et-Vilaine, G 153, $\mathrm{f}^{\circ} 76 \mathrm{r}^{\circ}$ ). Auteur de plusieurs ouvrages, il est membre de la censure royale (1736), de l'Académie royale des sciences et belles lettres de Berlin (1749) et de l'Académie française (1761). Ruffelet possède les quatre volumes de son Essai sur divers sujets de littérature.

$39.18 \%$ des 494 volumes de sa bibliothèque concernent l'ouverture, Arch. dép. de Loire-Atlantique, B 9989. Il est chanoine de Nantes de 1749 (Arch. dép. de Loire-Atlantique, $\mathrm{G} 852 \mathrm{f}^{\circ} 193 \mathrm{v}^{\circ}$ ) à 1784 (Arch. dép. de Loire-Atlantique, $3 \mathrm{E}$ 875). 
le mathématicien allemand Christian Wolff ${ }^{40}$. Par conséquent, cette ouverture se mesure presque exclusivement au travers de ses nombreux ouvrages d'histoire étrangère et de géographie.

Un premier constat s'impose : l'éclectisme de ses centres d'intérêt. Tous les continents sont en effet concernés et, en Europe, les principaux États retiennent son attention. Dans le détail, ce rayon semble vraiment novateur. En effet, on y note l'effacement de l'Italie et de la Méditerranée d'une manière générale, présentes à l'état résiduel. L'humanisme et la vieille idée de croisade ne font en effet plus recette. En revanche, l'Europe du nord - Angleterre bien sûr, qui fascine tant les élites de l'époque, mais aussi Suède, Allemagne ou Russie - l'intéresse particulièrement. Encore plus révélateur, notre chanoine ne dissimule pas un certain penchant pour l'exotisme : l'Amérique, l'Asie, l'Afrique, la Chine, le Japon, le Siam, le Mexique, l'Orénoque, le Paraguay, le Pérou représentent 15 titres pour 72 volumes. La bibliothèque de Ruffelet confirme donc l'inclinaison croissante du monde capitulaire pour l'étranger ${ }^{41}$ au sein d'un milieu ecclésiastique qui reste moins perméable que d'autres catégories sociales à cet aspect de l'histoire ${ }^{42}$. Une importante collection d'ouvrages de géographie complète ce rayon historique. Au-delà de sa dimension - on y observe de très nombreux ouvrages généraux -, ce qui le distingue, c'est la part offerte aux récits de voyages : une douzaine de titres pour 142 volumes ${ }^{43}$. Dans ce domaine, Ruffelet apparaît donc bien comme un enfant de son siècle. En ce temps qui se passionne pour les horizons lointains et connaît une progression considérable de ce type de publications ${ }^{44}$, il fait figure de chanoine d'avant-garde. Il possède quelques ouvrages que l'on trouve chez d'autres chanoines comme l'Utilité des voyages de Baudelot, le Nouveau voyage aux îles de l'Amérique du dominicain Labat, mais il y ajoute le Voyage au Cap de Bonne-espérance de La Caille, la Relation de l'Éthiopie de Labat et surtout le Voyage autour du monde de Bougainville.

Christophe-Michel Ruffelet est donc le propriétaire d'une bibliothèque atypique qui n'ignore aucun domaine et où sont accentués tous les signes de modernité que l'on peut observer chez certains de ses confrères.

40. Wolff (1679-1754) est aussi philosophe.

41. Plus visible du point de vue qualitatif que quantitatif : à Nantes, Rennes ou SaintPol-de-Léon l'intérêt pour l'Europe du Nord ou l'outre-mer s'affirme doucement.

42. QuÉNIART, Jean, Culture et société urbaines..., op. cit., p. 221-224.

43. Barrin (Arch. dép. de Loire-Atlantique, B 9972) de Nantes possède 10 titres de cette nature (11 volumes), Bossard (Arch. dép. d'Ille-et-Vilaine, 4 B 4817) de Rennes 9 (10 volumes), Le Sparfel (Arch. dép. du Finistère, 23 B 333) de Saint-Pol 7 (11 volumes) et Le Grand (Arch. dép. du Finistère, 23 B 369) de Saint-Pol 6 (11 volumes). Le Febvre de Nantes (Arch. dép. de Loire Atlantique, B 6907) ne possède que 3 titres... mais 24 volumes.

44. Roche, Daniel, Humeurs vagabondes. De la circulation des hommes et de l'utilité des voyages, Paris, Fayard, p. 19-48. 


\section{L'antiquaire et l'historien}

Incontestablement grand lecteur, assurément cultivé, propriétaire d'une bibliothèque ouverte aux évolutions intellectuelles de son temps, Ruffelet est aussi un auteur - historien de surcroît - engagé dans certains débats du temps et qui met sa plume au service des intérêts bretons. Qualités remarquées par la Société patriotique bretonne qui en 1782 distingue celui qui « depuis plus de vingt ans consacre ses talents à la gloire de sa patrie ${ }^{45}$ ". Très tôt il a en effet allié la passion des origines, en s'intéressant au temps les plus reculés de l'histoire bretonne, aux préoccupations les plus contemporaines, en réfutant des écrits hostiles aux droits de la province.

En 1762 et 1763 paraissent ses premiers travaux, consacrés au diocèse de Saint-Brieuc dont il veut faire connaître ce que l'" histoire ecclésiastique, naturelle, civile et politique offre de plus intéressant ". Dans Les Étrennes briochines, il publie d'abord des articles sur différentes paroisses, avant de se consacrer à des notices biographiques relatives aux saints, aux évêques, aux militaires, aux savants et aux écrivains originaires du diocèse. Ces écrits annoncent un ouvrage plus important, publié en 1771 : les Annales briochines ou abrégé chronologique de l'histoire ecclésiastique, civile et littéraire du diocèse de Saint-Brieuc. Ruffelet ne considérait cet ouvrage qui couvre la période entre « les temps qui ont précédé la venue de JésusChrist " et 1768 que comme "l'essai ou l'annonce d'un ouvrage beaucoup plus considérable "... qui ne verra jamais le jour. Les Annales montrent que les bases en étaient cependant jetées puisque de nombreuses notes sur des sujets divers - du christianisme des premiers temps à l'archéologie en passant par l'organisation politique du diocèse ou la présentation de ses principales cités - les accompagnent. Il n'a par ailleurs jamais cessé le travail. La parution des Annales le conduit en effet à poursuivre une correspondance engagée avec Pierre Corgne ${ }^{46}$, qui lui reproche de ne pas avoir tenu compte de certaines de ses analyses. Il est de plus l'auteur d'un Mémoire pour la collégiale de Saint-Guillaume de Saint-Brieuc : historique, fondations, notes ${ }^{47}$. Par la suite, vraisemblablement fin 1778 , il prend

45. Diplôme décerné par la Société patriotique bretonne à Ruffelet, 22 avril 1782. Reproduit dans DuBOIS DE LA VILLERABEL, Arthur, « Le chroniqueur Ruffelet »..., p. 148-149. Se voulant une sorte d'académie bretonne, cette société littéraire fondée en 1783 par le gouverneur de Rhuys Sérent regroupe des amateurs de l'histoire et des lettres bretonnes et se réunit au château de Keralier en Sarzeau. LA BORDERIE, Arthur de, « Le baron de Kerker et son château ", KERVILER, René, "Olivier Morvan (1754-1794) ", Revue de Bretagne et de Vendée, 1887, t. II, p. 321-336 et p. 337-354.

46. Né à Corlay vers 1700, Pierre Corgne est chanoine de Soissons. En 1734, il annule un acte de permutation conclu avec Jean-Guy de Durfort de Lorge d'Avaugour (Arch. diocésaines de Saint-Brieuc, 1 CH 10, papiers de l'abbé d'Avaugour), chanoine de la cathédrale de Saint-Brieuc. Docteur de Navarre, il est l'auteur de plusieurs travaux de natures théologiques et historiques.

47. Ce travail a été vendu à l'Hôtel des ventes de Saint-Brieuc le 21 février 1968 selon un petit catalogue de vente, Arch. diocésaines de Saint-Brieuc, dossiers du chanoine du Cleuziou, papiers provenant de la collection de M. Arthur du Bois de La Villerabel et des papiers Limon Belleissue. 
contact avec Ogée ${ }^{48}$ en lui proposant de rédiger l'article sur Saint-Brieuc et en lui envoyant une dissertation sur les origines de Carhaix. Si le second travail a été retenu dans le Dictionnaire historique et géographique de la province de Bretagne, il semble que le premier ait suscité quelques difficultés. En effet, en janvier 1779, Ogée envoie à Ruffelet le plan qu'il entend voir respecter, mais déplore en avril, après avoir lu la première notice du chanoine, que le travail ne soit pas suffisamment avancé. Par conséquent, il décide que "l'article ne sortira pas tout à fait de [ses] mains ", mais le presse toutefois de lui envoyer le reste de ses recherches tout en le dispensant du catalogue des évêques, déjà établi. En dépit de ces remarques, il semble que l'article sur la cité épiscopale peut lui être attribué : des passages entiers sont empruntés aux Annales briochines ${ }^{49}$; pour le reste, elles fournissent la trame des développements ${ }^{50}$. Ensuite, en 1780-1781, il échange avec le chevalier de Kerloury et Jacques Le Brigant ${ }^{51}$ au sujet du temple de Lanleff; en 1781, avec Pierre Millet ${ }^{52}$, recteur de Lamballe, au sujet de la collégiale. Dans un autre registre, il trouvera le temps de préparer un Programme d'exercices littéraires pour le Collège de Saint-Brieuc en 1782.

Parfaitement au fait des controverses historiques, Ruffelet s'immisce dans les débats sur les origines de la Bretagne et, " en tirant de l'obscurité plusieurs monuments qui ont échappé aux historiens de cette province ", il contribue à assurer "l'antiquité et la distinction de la nation bretonne ${ }^{53}$ ".

48. Jean-Baptiste Ogée est né en 1728. Fils d'un capitaine d'infanterie, il embrasse la carrière des armes avant de devenir ingénieur cartographe de la province de Bretagne à la suite d'une blessure. En 1753, il est inspecteur de la Route de Nantes à Ingrandes; en 1757, sous-ingénieur des Ponts et Chaussées et grands chemins de la province de Bretagne. Il réalise une Carte du comté nantais en 1768, l'Atlas itinéraire de Bretagne en 1769, une Carte géométrique de la province de Bretagne en 1771. En 1778-1780, il publie son Dictionnaire historique et géographique de la province de Bretagne dédié à la nation bretonne. Il meurt à Nantes en janvier 1789 .

49. Par exemple, les notions sur l'origine de Saint-Brieuc dans Ogée reprennent des passages des notes I, XIV et XV des Annales briochines; nombre des événements marquants de l'histoire du diocèse ne sont que la copie des notices annuelles des Annales.

50. Pour la description de l'économie et du commerce du diocèse par exemple, qui s'inspire de la Description générale du diocèse des Annales.

51. Avocat, philologue et grammairien, Jacques Le Brigant est né à Pontrieux en 1720 et mort à Tréguier en 1801. Collaborateur et ami de La Tour-d'Auvergne, il a beaucoup travaillé sur les origines de la Bretagne. Il est notamment l'auteur des Éléments de la langue des Celtes Comérites ou Bretons pour servir d'introduction à cette langue et par elle aux langues de tous les peuples parus en 1779. En 1781, il écrit à Ruffelet qu'il est le premier Briochin à avoir acheté ce livre; d'autres courriers portent sur le temple de Lanleff, les mines de Châtelaudren..

52. Pierre-François Millet, né à Saint-Alban le 5 avril 1735, est régent au collège de SaintBrieuc en 1761, chanoine de la collégiale Saint-Guillaume de Saint-Brieuc de 1768 à 1776, recteur de Saint-Mathieu de Moncontour de 1776 à 1779, de Notre-Dame et Saint-Jean de Lamballe de 1779 à 1791, puis de 1803 à 1814 après un séjour à Jersey : Arch. dép. des Côtes-d'Armor, G 47, $\mathrm{f}^{\circ} 394 \mathrm{r}^{\circ}$ et G 49, f $324 \mathrm{r}^{\circ}$; Arch. dép. d'Ille-et-Vilaine, fichier Berthelot du Chesnay.

53. Diplôme décerné par la Société patriotique bretonne à Ruffelet, 22 avril 1782. Reproduit dans DuBOIS DE LA VILLERABEL, Arthur, " Le chroniqueur Ruffelet... », art. cit., p. 148-149. 
Il faut entendre ici le terme monument dans son acception la plus large, incluant monuments, sculptures, inscriptions, monnaies, médailles. Il apporte en effet son éclairage sur l'utilisation ancienne du temple de Lanleff, s'efforce de dater un camp attribué à César sur le territoire de la paroisse de Pordic et donne de la publicité aux " antiquités découvertes depuis peu " (anciens murs, médailles, mosaïques) à Erquy ${ }^{54}$. Il réfléchit de plus aux origines des villes de Quintin, Lamballe, Moncontour, Chatelaudren, Saint-Brieuc, Carhaix ${ }^{55}$; brosse l'histoire du diocèse et du duché de Penthièvre ${ }^{56}$. C'est en définitive sur un triple héritage qu'il fonde l'histoire du diocèse de Saint-Brieuc : gaulois pour les origines du peuplement ${ }^{57}$, romain pour la civilisation ${ }^{58}$, breton pour la religion ${ }^{59}$.

Convaincu du rôle qu'ont pu jouer les Romains et les peuples armoricains avant eux, il entend démontrer la véracité de ce passé ancien en le dépouillant de toutes les légendes susceptibles de lui nuire, en se gardant de conclure prématurément si les preuves manquent. Ruffelet est en effet un esprit méticuleux qui entend prouver systématiquement ses dires à l'aide d'écrits ou de résultats de fouilles archéologiques. Ainsi, lorsqu'il étudie le réseau des anciennes routes romaines qui rayonnent à partir de Corseul il " ne parle que de celles dont on remarque encore aujourd'hui les vestiges ", en présentant le tracé et la technique de construction ${ }^{60}$. Examinant la fondation de la ville de Lamballe, il se refuse à l'attribuer aux Ambiliates dont parle César car certains auteurs les situent en Belgique et non en Armorique. Et de conclure : " Je ne crois pas les Lamballais descendus ni du tout parents des Ambiliates de César. Assez célèbres par euxmêmes, ils n'ont pas besoin de chercher dans une origine douteuse ${ }^{61}$. " En revanche, en ce qui concerne Carhaix, il critique vertement les positions de La Tour d'Auvergne qui attribue sa création au général romain Aetius au IV siècle. Selon lui, les origines de la ville doivent être recherchées beaucoup plus loin dans le passé, à l'époque "des Ossismiens, l'un des principaux peuples de l'Armorique ", tous " les précieux restes de l'antiquité ${ }^{62}$ " étant la marque de quatre ou cinq siècles de présence romaine. S'agissant du temple de Lanleff, il est aussi hésitant que Robien quelques années

54. RufFelEt, Christophe-Michel, Annales briochines..., notes XI, VII et X. Le temple d'Erquy a été découvert par Lobineau. Ce dernier est par ailleurs le premier à avoir fouillé Corseul. AUBERT, Gauthier, "À propos des débuts de l'archéologie en Bretagne (Corseul, Erquy et quelques autres lieux) ", Mémoires de la Société d'Histoire et d'Archéologie de Bretagne, t. LXXIV, 2002, p. 459-482.

55. RuffELET, Christophe-Michel, Annales briochines..., notes II, IV, V, VI, IX, XIV. OGÉE, Jean-Baptiste, Dictionnaire historique et géographique de la province de Bretagne, réédition A. Marteville, Rennes, 1843-1853, articles Carhaix et Saint-Brieuc.

56. RufFelet, Christophe-Michel, Annales briochines..., op. cit., note I et année 1696.

57. Ibidem, note I.

58. Par exemple, OGÉE, Jean-Baptiste, Dictionnaire historique..., op. cit., article Carhaix.

59. RuFFELET, Christophe-Michel, Annales briochines..., op. cit., note XIX.

60. Ibidem, note III.

61. Ibid., note V.

62. OGÉE, Jean-Baptiste, Dictionnaire historique..., op. cit., article « Carhaix ». 
auparavant ${ }^{63}$ puisque, si « la forme de ce monument jointe à une ancienne tradition appuyée au suffrage même de quelques savants, a fait croire que ce pouvait être les restes d'un ancien temple consacré autrefois aux idoles ", il affirme en 1771 qu'il faudrait attendre que " de nouvelles recherches et un examen encore plus exact nous mettent à même de le mieux connaître et de nous prononcer plus hardiment sur son ancienne destination $^{64}$ ". Il poursuit néanmoins lui-même des recherches dont témoigne sa correspondance avec le chevalier de Kerloury et Le Brigant.

Cette chasse aux légendes, si belles soient-elles, cette retenue, s'inscrivent dans une conception de l'histoire exigeante fondée sur la preuve. Ruffelet s'inscrit donc dans la tradition de l'histoire critique dont les fondements ont été posés par les mauristes. D'ailleurs, il possède un Dictionnaire de diplomatique dû à des bénédictins. Sa méthode d'exposition varie peu. Il commence par présenter le problème à résoudre : une thèse qu'il va invalider, une opinion reçue communément qu'il se propose d'étayer. Par exemple, il rappelle que la forme du camp de Pordic, sa situation, son nom, la tradition, l'histoire des guerres de César peuvent justifier son nom de " camp de César ${ }^{65}$ "; ou encore, que les inscriptions portées sur la châsse de saint Brieuc et la tradition font de ce dernier un évêque ${ }^{66}$. Dans un second temps, il développe son argumentation, fondée sur tel ou tel auteur ou fouille archéologique. En conclusion, il insiste sur le bénéfice à retirer de sa démonstration : datation mieux assurée d'un " des plus précieux et des plus anciens monuments d'antiquité du royaume de France " pour Pordic par exemple; ruine d'une considération sans fondement et nuisible à l'idée qu'elle prétend défendre, ou preuve d'une assertion héritée et donc renforcement d'une tradition locale pour d'autres sujets. Proche des positions de Lobineau - les Bretons trouvent " encore dans notre Armorique assez de vices et de pratiques supersticieuses à combattre ${ }^{67}$ »-, il n'en est toutefois pas prisonnier - les Bretons n'arrivent pas dans une région quasi déserte ${ }^{68}$ - et n'hésite pas à souligner ce qu'il considère comme des erreurs. Par exemple, selon Ruffelet, la Rheginea de la Table de Peutinger n'est pas Rohan dans l'évêché de Vannes mais Erquy dans celui de Saint-Brieuc ${ }^{69}$.

Dans son travail d'historien Ruffelet se montre à nouveau enfant de son siècle. En effet, la préface des Annales témoigne de sa foi en la fécondité du travail collectif; il se veut le maître d'œuvre d'une histoire du diocèse qui

63. AUBERT, Gauthier, Le président de Robien..., op. cit., p. 303.

64. RufFElET, Christophe-Michel, Annales briochines..., op. cit., note XI.

65. Ibidem, note VII

66. Ibid., note XVI.

67. Ibid., note XIX et QUÉNIART, Jean, "Les mauristes et l'historiographie bretonne ", dans : Tonnerre, Noël-Yves (dir.), Chroniqueurs et historiens de la Bretagne du Moyen Âge au milieu du XX $X^{e}$ siècle, Rennes, Presses Universitaires de Rennes, 2001, p. 119.

68. RufFElET, Christophe-Michel, Annales briochines..., op. cit., note I et QuÉnIART, Jean, "Les mauristes..." op. cit., p. 117.

69. RufFELET, Christophe-Michel, Annales briochines..., op. cit., note X. 
" demande trop de recherches, et dans des genres trop différents, pour pouvoir être bien exécutée par un seul auteur ${ }^{70}$ ". De plus, il connaît les sites archéologiques les plus récents. Mais on ne sait malheureusement pas si la description précise des lieux est le fruit d'une participation à des fouilles. Par ailleurs, son adhésion au plan proposé par Ogée pour élaborer la notice consacrée à Saint-Brieuc ne surprend pas : séparer l'histoire civile et politique du catalogue des évêques ${ }^{71}$ ne peut que permettre de gagner en rigueur historique. Enfin, il est convaincu que sa sensibilité aux traces du passé l'éloigne du commun, les paysans étant " plus jaloux de bonnes récoltes que de la conservation des anciens monuments ${ }^{72}$ ".

Le passé gaulois de la province, au-delà de son héritage romain, permet indirectement de dresser le portrait d'une Bretagne, d'un diocèse de SaintBrieuc en particulier, digne du reste du royaume. Cependant, c'est une Bretagne dotée de particularismes que Ruffelet défend dans les années $1765-1770^{73}$. En effet, passionné par les origines de sa province, il est aussi présent en son siècle. Pendant "l'affaire de Bretagne ", alors que les relations entre le Parlement et les États, d'une part, et la monarchie, d'autre part, sont particulièrement tendues ${ }^{74}$, Ruffelet est sollicité par l'avocat général Beaucours ${ }^{75}$ pour rédiger une critique des lettres envoyées par le contrôleur général Laverdy au Parlement. Ces lettres ont pour but d'établir « la pleine souveraineté du roi sur la province de Bretagne " et donc de justifier la levée d'un nouvel impôt sans le consentement des États. En 1767, alors que sa tâche n'est pas terminée, Ruffelet expose les axes de son argumentaire dans une lettre. Il y défend fermement les droits de la province, affirmant que " c'est la cause de la patrie que j'entreprends de plaider... ". À Laverdy qui affirme que la Bretagne n'est qu'un " démembrement, un fief détaché originairement de la Couronne " et qu'ainsi l'acte de 1532 est une réunion féodale, il rétorque que « l'origine et la source de la mouvance de Bretagne, il ne faut [...] aller la chercher que dans le besoin de protection, dans le respect dû à une puissance supérieure, dans les efforts faits pour assujettir une nation sur laquelle on ne peut jamais conserver qu'une simple reconnaissance de supériorité... ». Selon lui l'acte de 1532 ne peut

70. Ibidem, avertissement.

71. Du boIS DE LA VILLERABEL, Arthur, "Le chroniqueur Ruffelet »..., op. cit., p. 179.

72. RufFELET, Christophe-Michel, Annales briochines..., op. cit., note VII.

73. Du bois DE LA VILLERABEL, Arthur, "Le chroniqueur Ruffelet »..., op. cit., p. 383-423.

74. "L'affaire de Bretagne " s'inscrit dans un contexte plus large d'opposition parlementaire à l'échelle du royaume. Dans la province, depuis 1762 et le conflit lié à l'instauration d'un nouveau vingtième, la monarchie est en but à l'opposition des États de Bretagne... qui obtiennent rapidement le soutien du Parlement. L'animosité entre le commandant en chef, d'Aiguillon, et le procureur général du Parlement, La Chalotais, durcit le conflit. Lorsque Ruffelet écrit à Beaucours, le Parlement, démissionnaire, a été remplacé par le " bailliage d'Aiguillon " (mai 1765) et La Chalotais est emprisonné (depuis novembre 1765).

75. Hyppolite-Louis-Marie Loz de Beaucours, né en 1746 à Rennes, est conseiller au Parlement de Bretagne avant de devenir avocat général de 1779 à 1789. Levot, Pierre, Biographie bretonne..., op. cit., p. 362-363. 
donc être qu'une union libre consentie par la Bretagne afin de "prévenir ses malheurs " à une époque où elle était convoitée par les maisons de France, d'Espagne, de Penthièvre, de Rohan, de Savoie. Dans un autre écrit intitulé Réponse au Mémoire historique et critique sur les droits de souveraineté relativement au Traité de Bretagne, il complète ses analyses en démontrant que les nouveaux droits de traite en Bretagne ne sont pas domaniaux et ont " toujours dépendu du consentement libre de la nation ". Ces " subventions"-accordées pour des motifs imprévus, et donc révocables - relèvent des " droits et privilèges " de la province qui " furent conservés dans leur entier " et " devinrent même plus sacrés, plus inviolables par cette union ". Relayant ici une position classique de la noblesse, il s'engage dans un débat où l'interprétation de l'histoire de la Bretagne est devenue un enjeu. En effet, au début du siècle déjà, la monarchie et les États avaient échangé des arguments similaires ${ }^{76}$. Quittant le domaine juridique, il fournit ensuite - marque du temps - des arguments économiques imprégnés d'un libéralisme commercial cher aux élites éclairées du royaume ${ }^{77}$ : l'augmentation des taxes sur les marchandises peut provoquer la baisse de la consommation et donc l'affaiblissement du royaume. Selon Ruffelet, une taxation trop élevée à l'exportation renchérit les produits français par rapport aux productions étrangères et entraîne la diminution des entrées d'argent " qui font la richesse de l'État ". Par ailleurs, le ralentissement des exportations fragilise l'agriculture et l'industrie, empêchant à terme le peuple de se procurer des subsistances et de payer l'impôt. En définitive, selon Ruffelet, les États, en discutant les nouvelles impositions, choisissent le camp du progrès, des réels intérêts du royaume, et ne se complaisent pas dans une simple défense de privilèges.

Ruffelet est donc un esprit cohérent tout entier attaché à construire une certaine image de la Bretagne. Sa province est une Bretagne ancienne, aux racines au moins aussi lointaines que celles du royaume; c'est une Bretagne indépendante et maîtresse de son destin, même dans les circonstances les plus délicates; c'est, fort logiquement, une Bretagne qui doit être ménagée par le souverain et qui mène une lutte légitime contre les pouvoirs accrus de la monarchie au détriment de ses libertés. Les Lumières de Ruffelet conjuguent ainsi les libertés de la province avec la promotion de son passé le plus ancien et les théories économiques en vogue.

Tous ces travaux s'appuient sur un solide rayon de livres consacrés à la province, tout en étant certainement aussi, pour une part, à l'origine de sa constitution. Peu de domaines échappent à sa curiosité; mais l'histoire,

76. Déjà entre 1702 et 1706, l'intendant et les États contrôlés par la noblesse s'affrontent autour de ces questions. Plus tard, au moment de l'affaire Pontcallec, la noblesse fait l'amalgame entre ses prérogatives et les privilèges de la province. À partir de 1749 , l'établissement du vingtième provoque à nouveau des débats autour des droits de la Bretagne.

77. Depuis le milieu du siècle, les physiocrates, sous l'impulsion de François Quesnay, critiquent la logique mercantiliste et militent pour la liberté du commerce, partant du principe qu'il faut laisser faire "les lois de la nature". 
le droit et dans une moindre mesure les affaires politiques récentes lui sont plus familières.

Tableau 5 - La Bretagne dans la bibliothèque de C.-M. Ruffelet

\begin{tabular}{|l|c|c|}
\hline & Volumes & Titres \\
\hline Histoire profane & 35 & 13 \\
Histoire ecclésiastique & 7 & 2 \\
Dictionnaires historiques & 4 & 1 \\
Droit civil & 21 & 13 \\
Politique & 10 & 4 \\
Dictionnaires littéraires & 7 & 3 \\
Grammaire & 1 & 1 \\
Liturgie/encadrement & 4 & 3 \\
Dévotion & 1 & 1 \\
Statuts diocésains & 1 & 1 \\
Agriculture & 2 & 1 \\
Armorial & 1 & 1 \\
Total & 94 & 45 \\
\hline
\end{tabular}

Une nouvelle fois, Ruffelet se démarque de ses confrères. Certes, 9 des 13 bibliothèques de chanoines inventoriées à Nantes, Rennes et Saint-Polde-Léon entre 1766 et 1785 abritent des livres consacrés à la Bretagne, mais celle de Christophe-Michel surpasse les mieux pourvues : 24 volumes (11 titres) chez le Rennais Bossart, 17 volumes chez les Léonards Le Grand et Le Sparfel. En ce qui concerne l'histoire seule, l'écart est encore plus grand. Il fait donc preuve d'une sensibilité pour l'histoire bretonne que l'on ne retrouve pas chez ses confrères et qui le rapproche de certaines composantes des élites de la province-parlementaires et juristes notamment ${ }^{78}$. À des ouvrages également lus par certains de ses confrères comme les Histoires de Bretagne de Bertrand d'Argentré (3 mentions) et Morice (5 mentions), il ajoute l'Histoire de Bretagne de Lobineau, celle de Desfontaines, celle d'Astier. La présence du Dictionnaire historique et géographique de la province de Bretagne d'Ogée est quant à elle assez exceptionnelle. L'Histoire des Celtes et l'Histoire de l'établissement des Bretons dans les Gaules, auxquelles ont peut joindre des dictionnaires de langue bretonne ou celtique, rappellent son intérêt pour les origines de la Bretagne. L'Histoire de la réunion de la Bretagne à la France et les ouvrages de droit - dont plusieurs Coutumes, les Édits et déclarations du Parlement de Bretagne, le Journal du Parlement de Bretagne - rappellent que l'histoire est en partie une histoire de juristes "dominée par la polémique autour des privilèges provinciaux $^{79}$ ", polémique à laquelle Ruffelet lui-même a pris part. D’ailleurs, les

78. QUÉNIART, Jean, Culture et société urbaines..., op. cit., p. 326-327; AUBERT, Gauthier, «Une ville sans "histoire" au XVIII ${ }^{\mathrm{e}}$ siècle : Rennes ", dans CROIX, Alain, LESPAGNOL, André, ProvosT, Georges, Élise, Éducation, Lumières... Histoires culturelles de la France (1500-1830). En l'honneur de Jean Quéniart, Rennes, Presses Universitaires de Rennes, 1999, p. 264-265.

79. AUBERT, Gauthier, Le président de Robien..., op. cit., p. 265. 
affaires politiques très récentes de la province ne sont pas absentes de sa bibliothèque comme en témoignent par exemple les Preuves de la souveraineté du roi en Bretagne, les Affaires de Bretagne en 1763 et suivantes, le Règlement des trois États en Bretagne de 1787...

\section{Le chanoine}

L'importance de sa bibliothèque et la reconnaissance intellectuelle dont jouit le personnage conduisent à s'interroger sur sa personnalité. À première vue, rien ne semble distinguer Ruffelet du chanoine breton ordinaire. Certes, alors qu'une écrasante majorité de ses confrères est noble, il est issu d'une famille roturière, faisant ainsi partie de cette minorité de chanoines non nobles du XVIII ${ }^{\mathrm{e}}$ siècle ${ }^{80}$. Mais la famille Ruffelet, de bonne bourgeoisie, est depuis des générations impliquée dans la gestion des affaires de Saint-Brieuc et le père de Christophe-Michel, Alain-François, en est même le maire en 1727. Par conséquent, Ruffelet ne semble pas atypique au sein de l'univers capitulaire. Il constitue même une nouvelle preuve de la captation des bénéfices urbains majeurs par les élites urbaines. En effet, en Bretagne, pour nombre de familles, une place dans un chapitre cathédral est associée à d'autres fonctions éminentes dans les sociétés locales. Ainsi plus de la moitié des chanoines dont la fonction du père est connue sortent du monde militaire, du milieu des cours souveraines, des présidiaux ou du négoce.

La présence de Ruffelet dans la cathédrale tend donc à montrer qu'ici aussi, quoique à un niveau inférieur, le canonicat est associé au dispositif de notabilité local. Du côté paternel, plusieurs ont en effet occupé des fonctions en vue dans la cité briochine. Sans remonter jusqu'à Salomon, sénéchal des régaires, anobli en août 1614 par Louis XIII pour services rendus pendant la Fronde, on trouve dans les différentes branches de la famille plusieurs députés de la ville de Saint-Brieuc aux États de Bretagne, des procureurs syndics des bourgeois de la ville ainsi que des avocats au Parlement ${ }^{81}$. En ce qui concerne la branche des Ruffelet du Chalonge, l'ascension semble plus tardive puisqu'elle s'affirme vraiment avec Gilles et Alain-François, l'oncle et le père de Christophe-Michel. Le premier est receveur du chapitre cathédral de Saint-Brieuc en 1718. L'un de ses fils Pierre sera gendarme de la Garde du roi, capitaine de cavalerie, chevalier de SaintLouis; un autre Pierre-Maurille, avocat au Parlement et subdélégué de l'intendant. Le second épouse en 1716 Hélène Lymon de Belle-Issue, fille de Pierre Lymon, lieutenant des régaires de Saint-Brieuc - et sœur de FrançoisMichel maire de Saint-Brieuc, procureur du roi à l'Amirauté, avocat au Parlement puis substitut du procureur au Parlement, et de Pierre-Michel,

80. 70 \% des chanoines bretons du XVIII ${ }^{\mathrm{e}}$ siècle sont issus de la noblesse. À Saint-Brieuc, en 1790, sept des dix chanoines dont on connaît l'origine sociale sont des nobles.

81. Pour toutes les informations de natures biographiques et familiales, voir les schémas généalogiques en annexes. 
avocat à la cour. La famille maternelle n'est donc pas en reste, d'autant qu'Hélène est la nièce de Pierre Chassin, " ancien bourgeois et maire héréditaire et en titre de la ville de Quintin, député aux États de Bretagne au dit Saint-Brieuc ". La qualité des parrains des enfants Ruffelet et Lymon témoigne aussi de l'intégration de ces familles dans la bonne société briochine. Deux oncles d'Alain-François ont pour parrains au milieu du $\mathrm{XVII}^{\mathrm{e}}$ siècle le procureur fiscal et le syndic des bourgeois de Saint-Brieuc. Michel-Charles et Yves Lymon, deux des frères d'Hélène Lymon, ont respectivement pour parrains Michel Chassin, procureur fiscal de Quintin, et un alloué de Corlay. Pierre Ruffelet, le cousin militaire de Christophe-Michel, est quant à lui en 1726 le filleul de Pierre-Guillaume de la Vieuville, l'évêque de Saint-Brieuc.

En dépit de ces fonctions et de ces fréquentations, il reste difficile de saisir la stratégie mise en œuvre par Christophe-Michel Ruffelet pour accéder aux stalles de la cathédrale en 1789. Il obtient en effet sa prébende alors qu'il a 64 ans $^{82}$. Or, ses confrères briochins en place au début de la Révolution ont pris possession à 33 ans en moyenne; à l'échelle de la Bretagne, cet âge moyen est de 33,5 ans sur le siècle. Pourquoi cet âge tardif? La véritable originalité de Ruffelet semble être son long séjour à la collégiale Saint-Guillaume entre $1771^{83}$ et 1789 . Saint-Brieuc est en effet avec Nantes la seule ville épiscopale bretonne de " duo ", c'est à dire caractérisée par la coexistence d'une cathédrale et d'une collégiale. Sa trajectoire n'est évidemment pas isolée : au cours du XVIII siècle, onze Briochins et dix Nantais passent de la collégiale à la cathédrale ${ }^{84}$. À la veille de la Révolution six autres Briochins ont d'ailleurs suivi cette trajectoire, mais Ruffelet détient le plus long des séjours en collégiale. Il est cependant malaisé d'expliquer ces itinéraires. On peut certainement y lire le poids des préoccupations financières. La prébende - le revenu attaché au canonicat de la cathédrale rapporte en effet davantage que celui de la collégiale : 870 livres contre 333 à la veille de la Révolution à Saint-Brieuc ${ }^{85}$. Mais, cette

82. Arch. dép. des Côtes-d'Armor, G 83, $\mathrm{f}^{\circ} 65 \mathrm{v}^{\circ}$.

83. C'est Sigismond Ropartz qui indique la date du 27 novembre 1771 dans l'édition de 1851 des Annales briochines. L'acte de prise de possession ne figure pas dans les registres d'insinuations ecclésiastiques du diocèse. Cependant, le registre couvrant la période 17691774 indique qu'il obtient une lettre de prêtrise le 20 mai 1771 et qu'il fait partie des chanoines présents lors de la prise de possession de son confrère Louis-François Despons le 7 avril 1775 (Arch. dép. des Côtes-d'Armor, G 48, $\mathrm{f}^{\circ} 185 \mathrm{r}^{\circ}$ et $\mathrm{G} 49, \mathrm{f}^{\circ} 159 \mathrm{r}^{\circ}$ ). Par ailleurs, une mention de 1779 indique qu'il a obtenu des provisions royales le 6 octobre 1771 (Arch. dép. des Côtes-d'Armor, G 51, f ${ }^{\circ} 58$ v $^{\circ}$ ).

84. Soit autour de $10 \%$ des effectifs des deux compagnies.

85. Respectivement Arch. nat., G $880^{1}$, état présenté par le chapitre cathédral de SaintBrieuc à l'Assemblée générale du clergé de France à fins d'indemnités et de secours, 1786 et NiÈres, Claude (dir.), Histoire de Saint-Brieuc, Toulouse, Privat, 1991, p. 76. A Nantes, la prébende de Notre-Dame rapporte 657 livres alors que celle de la cathédrale en rapporte 2200. LA NiCOLLIÈRE, Stéphane de, Église royale et collégiale de Notre-Dame de Nantes, monographie historique et archéologique, Nantes, Forest et Grimaud, 1865, p. 203; Arch. dép. de Loire-Atlantique, Q 33, déclaration de revenus du chapitre cathédral de Nantes, 1790. 
somme restant inférieure aux revenus que procurent certaines cures du diocèse ${ }^{86}$, les questions de prestige peuvent tout autant y jouer leur rôle. Sans être aussi définitif que certains pour qui « le camail de Saint-Guillaume était l'acheminement ordinaire à celui de la cathédrale, supérieur en dignité $^{87}$ ", cela nous renvoie incontestablement au profil des deux compagnies et à leur insertion dans le jeu social local. Les chanoines de la collégiale étant malheureusement très mal connus ${ }^{88}$, nous sommes contraints de nous contenter de ces hypothèses.

Tout au plus savons-nous que la famille de Christophe-Michel entretient des liens étroits avec l'univers capitulaire. Son grand-oncle JeanFrançois Ruffelet est chanoine de Saint-Guillaume au début du siècle, un grand-oncle de sa mère, Pierre Lymon de la Vieuxville est chanoine de la cathédrale à la fin du XVII ${ }^{\mathrm{e}}$ siècle. L'une de ses tantes est par ailleurs la bellesœur de Guillaume Le Floc'h, chantre et chanoine de la cathédrale ${ }^{89}$, et les Lymon comptent certains d'entre eux au nombre des chanoines de la collégiale de Quintin ${ }^{90}$. Insérée dans un tissu capitulaire unissant la cathédrale de Saint-Brieuc et les collégiales de Saint-Brieuc et de Quintin, la famille de Ruffelet n'est de plus pas inconnue dans l'administration ecclésiastique : son oncle Gilles Ruffelet occupant le poste de receveur du chapitre cathédral au début du XVIII ${ }^{\mathrm{e}}$ siècle, son grand-père Pierre Lymon de Belle-Issue celui le lieutenant des régaires. Autant d'arguments qui peuvent servir les desseins d'un clerc ambitieux. Ce dont on peut en effet être certain, c'est que Christophe-Michel doit se sentir à l'étroit à l'ombre de la collégiale. En 1775, les rumeurs de départ de Jean-Joseph Du Fau lui font espérer un canonicat à la cathédrale. Il établit donc de bonne relations avec lui pendant que son cousin, gendarme de la Garde du roi, sollicite les membres d'un réseau auquel appartiennent le duc de Chartres, son précepteur l'abbé de Bonrepos, Soubize, capitaine-lieutenant des gendarmes de la Garde du roi. Malheureusement Du Fau conserve son bénéfice ${ }^{91}$. Quatre ans plus tard, Christophe-Michel semble manifester à nouveau le

86. Berthelot DU ChESNAY, Charles, Les prêtres séculiers en Haute-Bretagne au XVII ${ }^{e}$ siècle, Rennes, Presses Universitaires de Rennes, 1974, p. 647-651.

87. Du BoIS DE LA VILLERABEL, Arthur, "Le chroniqueur Ruffelet ", op. cit., p. 139.

88. Un sondage dans les registres d'insinuations ecclésiastiques permet de reconstituer le chapitre de Saint-Guillaume lors de l'arrivée de Christophe-Michel. Le portrait de groupe qui s'en dégage est proche de celui des chapitres des cathédrales. Ruffelet y rencontre en effet au moins onze prêtres parmi les seize chanoines identifiés (la collégiale abrite théoriquement 20 chanoines). Par ailleurs, les huit dont l'origine géographique est connue sont originaires du diocèse de Saint-Brieuc, dont au moins six de la cité épiscopale.

89. De 1713 (Arch. dép. des Côtes-d'Armor, G 37, f 22 r $^{\circ}$ ) à 1749 (Arch. dép. des Côtesd'Armor, G 42, p. 586).

90. Voir annexes 1 et 2 et Arch. mun. de Saint-Brieuc, 10 Z 3, fonds Lymon de La Belleissue. Manuscrit de François Lymon de Belleissue : chronique familiale (XVIII ${ }^{\mathrm{e}}$ siècle avec rétrospectives jusqu'au $\mathrm{XV}^{\mathrm{e}}$ siècle).

91. Du BoIS DE LA VILLERABEL, Arthur, "Le chroniqueur Ruffelet ", op. cit., p. 139-143. Du Fau est chanoine de la cathédrale de 1770 (Arch. dép. des Côtes-d'Armor, G $48 \mathrm{f}^{\circ} 94 \mathrm{r}^{\circ}$ ) à 1776 (Arch. dép. des Côtes-d'Armor, G 49 f $^{\circ} 330 \mathrm{v}^{\circ}$ ). 
souhait de quitter la collégiale : sa démission du 5 octobre 1779 ne sera cependant pas suivie d'effet ${ }^{92}$.

Chanoine sur le tard à 46 ans, Ruffelet se distingue des autres prébendés, en général déjà pourvus depuis de longues années. Est-ce à dire qu'à la différence de la majorité de ses confrères bretons, il aurait occupé d'autres bénéfices ${ }^{93}$ pendant les vingt-deux années qui séparent son accession à la prêtrise et son entrée à Saint-Guillaume? Les aléas des débuts de sa carrière ecclésiastique sont malheureusement très mal connus et entre ces deux dates une large zone d'ombre le dérobe à l'analyse. Tout au plus peut-on conclure qu'il fait partie de ces chanoines bretons qui n'ont ni reçu une formation parisienne ${ }^{94}$, ni obtenu de grades universitaires ${ }^{95}$. En effet, après des études de théologie au collège de Saint-Brieuc, il reçoit la tonsure et les quatre mineurs le 18 juin 1747 puis la prêtrise le 20 septembre 1748 à Saint-Brieuc ${ }^{96}$. Il est tout à fait possible que Christophe-Michel ait en fait vécu parmi les siens et se soit entièrement consacré à des travaux historiques tout en constituant son imposante bibliothèque. C'est pendant cette période qu'il publie les Étrennes briochines, rédige ses écrits politiques et prépare les Annales briochines. Il a d'ailleurs tiré profit de la curiosité intellectuelle développée dans sa famille maternelle puisque les Annales briochines bénéficient des travaux de son oncle François-Michel Lymon ${ }^{97}$. Dégagé des contingences matérielles et baignant dans un milieu favorable à l'étude, on tiendrait donc là le portrait d'un prêtre érudit qui trouve dans de tardifs canonicats les conditions propices à la poursuite d'un travail intellectuel d'envergure. "Bibliomanie chez Prud'homme ", villégiature chez sa cousine, la marquise de la Rivière, et quelques séjours parisiens agrémentent cette existence dédiée au travail intellectuel ${ }^{98}$. Faute de documentation, on ne sait comment il assume les devoirs de ses prébendes suc-

92. Arch. dép. des Côtes-d'Armor, G 51, $\mathrm{f}^{\circ} 58 \mathrm{v}^{\circ}$

93. Si l'on ne considère que les chanoines des cathédrales bretonnes, le canonicat est en règle générale leur premier et parfois seul bénéfice d'envergure. En effet, pour plus de la moitié d'entre eux il est une fin en soi, pour un tiers un aboutissement (après un premier canonicat pour la moitié), pour $10 \%$ un point de départ et pour $5 \%$ une étape entre deux bénéfices. Cette réalité mériterait d'être vérifiée pour les chanoines des collégiales. Quelques exemples briochins laissent penser que l'hypothèse est fondée.

94. Alors que chez les Lymon il existe une tradition des études lointaines. Il est en effet courant d'envoyer les garçons suivre des études de droit à Caen ou à Nantes. Arch. mun. de Saint-Brieuc, 10 Z 3, fonds Lymon de La Belleissue. Manuscrit de François Lymon de Belleissue : chronique familiale (XVIII ${ }^{\mathrm{e}}$ siècle avec rétrospectives jusqu'au $\mathrm{Xv}^{\mathrm{e}}$ siècle).

95. Nombre de chanoines fréquentent en effet les séminaires et l'université parisiens. Par ailleurs, 44 \% des chanoines bretons sont gradués lors de leur prise de possession; si l'on ajoute ceux qui poursuivent leurs études après être entrés dans un chapitre, on atteint une proportion de l'ordre de la moitié.

96. Arch. dép. des Côtes-d'Armor, G 42, p. 387 et $489 ; \mathrm{G} 43, \mathrm{f}^{\circ} 22 \mathrm{v}^{\circ}$.

97. LAMARE, Jules, Histoire de Saint-Brieuc, SECN, 1922, p. 190; [F.-M. LYMON], "Journal historique et domestique d'un magistrat breton (1694-1765) ", édité par A. DuBoIs de LA Villerabel, Mémoires de la Société Archéologique et Historique des Côtes du Nord, $2^{\mathrm{e}}$ série, t. II, 1885-1886, p. 152-199.

98. Dubois de LA VILLERABEL, Arthur, "Le chroniqueur Ruffelet ", op. cit. 
cessives. Sa présence lors des prises de possessions de confrères à la collégiale, son assiduité pendant la rigoureuse puis dans les semaines qui suivent à la cathédrale laissent cependant entrevoir un chanoine assidu ${ }^{99}$. Quelques rares documents montrent qu'il ne se dérobe pas à ses obligations administratives : en 1783-1784 il assume, à son tour, la charge de commissaire chargé de vérifier les comptes présentés par le comptable de la collégiale $^{100}$. Il convient cependant de demeurer prudent. En effet, l'exemple d'un autre chanoine - le Rennais Du Breil de Pontbriand - accaparé par des tâches d'archiviste et d'historien, délaissant de ce fait stalle et salle capitulaire ${ }^{101}$, montre que les devoirs de la prébende ne sont pas toujours compatibles avec un travail intellectuel soutenu.

Afin de parfaire la connaissance du personnage, les secteurs modernes de la bibliothèque de Ruffelet et ses ambitions d'historien doivent être rapprochés d'autres indices de modernité ${ }^{102}$. Il possède en effet " une collection de minéraux et coquillages ", une collection de soixante-neuf tableaux " de différentes grandeurs peu estimés des connaisseurs et représentant différents paysages et les portraits de plusieurs grands hommes tels que Rousseau, La Fontaine... ", des cartons d'estampes de l'imprimerie de Panckoucke, des figures chinoises en terre et en porcelaine et quatre tableaux chinois. Par ailleurs, son intérêt pour les horizons proches ou lointains est confirmé par " une carte générale de la province de Bretagne levée géométriquement et assujettie aux observations astronomiques de Mrs de l'Académie royale des sciences en 1775 ", des cartes " célestes et ter-

99. Arch. dép. des Côtes-d'Armor, G 48, 49, 5051 et 52, registres d'insinuations ecclésiastiques (1769-1790); G 83, registre des délibérations du chapitre cathédral de SaintBrieuc, 1780-1790. La rigoureuse est le stage obligatoire qu'effectue le nouveau chanoine afin de se familiariser avec les devoirs temporels et spirituels de son bénéfice. Pendant cette période plus ou moins longue selon les lieux - six mois à Saint-Brieuc - il est obligé de résider dans la ville épiscopale, d'assister à un certain nombre offices... et ne touche pas les revenus de sa prébende.

100. Arch. dép. des Côtes-d'Armor, G 131, comptes de la collégiale Saint-Guillaume de Saint-Brieuc, 1762-1784.

101. Pontbriand semble en fait être l'archétype du chanoine plus intéressé par les travaux intellectuels que par les devoirs de la prébende. Il mène en réalité une double carrière de chanoine et d'intellectuel. Doté de sa prébende en 1728, il se voit confier le classement des archives du chapitre et des États dès 1730. Ces travaux achevés, il se lance dans l'analyse des délibérations des États et entreprend la rédaction d'une Histoire générale des États de Bretagne entre 1567 et 1754, œuvre de longue haleine qu'il n'achèvera pas. Il a également trouvé le temps de rédiger les Nouvelles vues sur le système de l'univers en 1751 et un Essai de grammaire française en 1754. Au cours de ces années, il se distingue par son remarquable absentéisme. Par exemple, au cours de l'année capitulaire 1746-1747 il n'assiste pas à un seul chapitre (Arch. dép. d'Ille-et-Vilaine, G 204) et n'est présent au chœur qu'aux matines des 12 octobre 1746, 13 mars et 6 juin 1747, à la grandmesse du $1^{\text {er }}$ avril 1747 (Arch. dép. d'Ille-et-Vilaine, G $261 \mathrm{H}$ ). Une analyse rapide des registres de pointes des années 1734-1739 confirme ce désintérêt (Arch. dép. d'Ille-etVilaine, G $261 \mathrm{H}$ ).

102. Arch. dép. des Côtes-d'Armor, 1 Q 517, inventaire des biens de Ruffelet, 12 messidor II. 
restres ", " une sphère avec un globe ", huit baromètres et thermomètres. On peut d'ailleurs se demander s'il n'a pas installé une sorte de petit cabinet de curiosité - ou peut-être de physique - dans une petite pièce car l'exécuteur testamentaire Louis-Jean Prud'homme ${ }^{103}$ évoque un local où devaient se trouver "plusieurs instruments de physique et de mathématiques " ainsi que la plupart des objets évoqués ci-dessus ${ }^{104}$. De plus, il semble avoir mené de nombreux travaux scientifiques puisqu'il aurait détruit peu de temps avant sa mort " les résultats d'expériences scientifiques auxquelles il avait passé beaucoup de temps ${ }^{105}$ ". Dans un autre registre, il dispose d'un bidet et d'une pendule, indice d'une réelle attention à un certain confort matériel ${ }^{106}$. Il appartient par ailleurs à la Société d'agriculture de Bretagne, à laquelle sont liés quelques autres prébendés ${ }^{107}$. Ruffelet est donc bien l'archétype du chanoine ouvert à la culture de son temps... mais ne voulant surtout pas rompre avec les cadres sociaux, politiques, ecclésiaux traditionnels ${ }^{108}$. Le personnage n'est d'ailleurs peut-être pas si isolé que cela. En effet, dans toutes les compagnies bretonnes, une minorité de chanoines tente de maintenir l'Église au contact des évolutions majeures. L'intérêt manifesté dans tous les chapitres par certains chanoines pour les aspects les plus modernes de la culture de la fin du XVIII ${ }^{\mathrm{e}}$ siècle ne les conduit cependant pas à se distinguer de la masse de leurs confrères pendant la Révolution. En effet, seuls cinq s'engagent dans le camp de la

103. Louis-Jean Prud'homme dirige la principale imprimerie briochine de 1778 à 1811 . Il imprime pour le roi, l'évêché, la ville et le collège, et possède une importante librairie. Il semble être un proche de Ruffelet.

104. Arch. diocésaine de Saint-Brieuc, M 51, legs chanoine Ruffelet (bibliothèque), 1806-1883.

105. Selon S. Ropartz, Ruffelet, Christophe-Michel, Annales briochines..., op. cit., réédition 1851, p. XVI.

106. CROIX, Alain, « Le clergé paroissial, médiateur du changement domestique? Quelques remarques méthodologiques, quelques résultats ", Clercs et changement matériel. Travail et cadre de vie, $X V^{e}-X X^{e}$ siècles, Annales de Bretagne et des Pays de l'Ouest, t. 94, 1987, p. 465. Partout, les pendules comme les bidets apparaissent chez les chanoines de la fin de l'Ancien Régime.

107. Fondée en 1757 , elle se propose, conformément aux vœux des physiocrates, de développer les prairies artificielles, de vulgariser les méthodes de culture les plus productives, de favoriser la liberté du commerce des grains, d'encourager les défrichements. MorICEAU, Jean-Marc, "Sociétés d'agriculture ", BÉLY, Lucien (dir.), Dictionnaire de l'Ancien Régime, p. 1169 et VILLIERS, L. de, "Histoire de la Société d'agriculture, de commerce et des arts, établie par les États de Bretagne (1757) ", Association Bretonne, t. XVI, 1897, p. 312-352.

108. D'ailleurs, cette position fait écho à certains éléments de sa bibliothèque. Il possède ainsi un large rayon d'ouvrages de controverse : livres anti-protestants (Histoire des variations des Églises protestantes, Lettres d'un docteur catholique à un protestant sur les points de controverse...), anti-jansénistes (Instructions pastorales de Mgr de Cambray contre le jansénisme, Dissertation theologica contra jansenisma...), anti-philosophiques (Censure de la faculté de Paris contre Émile, Erreurs de Voltaire, Vérité de la religion prouvée à un déiste, le déisme réfuté par lui-même, Dictionnaire antiphilosophique, Procès divers contre les matérialistes...). Ce rayon représente $22 \%$ des 1482 titres religieux de sa bibliothèque, juste derrière les ouvrages de dévotion et de spiritualité (26\%). Par ailleurs, dans le domaine historique il fait plus confiance au dictionnaire de Moreri qu'à celui de Bayle. 
Révolution. Quant à Ruffelet, il dépose fin 1790 - alors que la législation ne l'y contraint pas - sa lettre de refus de serment à la Constitution civile du clergé. Emprisonné, libéré en mars 1795, il disparaît de la documentation pour réapparaître en qualité de chanoine du chapitre concordataire de Saint-Brieuc.

Christophe-Michel Ruffelet est aussi un représentant de cette minorité de chanoines intellectuels d'envergure. Certes, beaucoup de chanoines possèdent des bibliothèques, beaucoup sont docteurs ${ }^{109} \ldots$ mais seuls quelques-uns se lancent dans des travaux historiques. Il prend ainsi place aux côtés de quelques rares confrères aux ambitions différentes : si le Malouin Porée du Parc s'en tient à sa ville ${ }^{110}$, le Dolois Déric prend pour objet d'étude la Bretagne ecclésiastique ${ }^{111}$ et le Rennais Du Breil de Pontbriand met ses compétences au service des États de Bretagne ${ }^{112}$. Beaucoup moins nombreux que dans les chapitres de Guyenne ou du Nord, ils annoncent les chanoines érudits du XIX ${ }^{e}$ siècle et de la première moitié du xx ${ }^{\mathrm{e}}$ siècle. Ruffelet, Déric, Porée et Du Breil (auxquels on pourrait joindre Moreau ou Chapelain ${ }^{113}$ ) sont comme ces derniers des locaux, des personnalités enracinées, et non des greffes extérieures. Le milieu urbain et le clergé bretons engendrent donc ce type d'érudits qui sont autant de maillons de la longue chaîne des chanoines intellectuels qui mériterait d'être étudiée. Tout comme mériterait d'être approfondie la question de l'absence - à une exception près - de rédaction d'histoires des compagnies bretonnes au cours du XVIII ${ }^{\mathrm{e}}$ siècle.

Plus largement, Ruffelet renvoie à un milieu assez mal connu : celui des cercles intellectuels bretons de la fin de l'Ancien Régime, puisque ses travaux l'inscrivent dans un réseau qui s'élargit avec le temps. En relation étroite avec les Briochins Lymon de La Belleissue, La Villerabel et Armez ${ }^{114}$,

109. Au cours du XVIII ${ }^{\mathrm{e}}$ siècle, 40 \% des nouveaux chanoines des cathédrales bretonnes sont docteurs.

110. PorÉE du PARC, René-Joachim, Histoire de la seigneurie ecclésiastique en la ville de Saint-Malo et dépendances dans la paroisse de Saint-Servan, dédiée à Monseigneur l'évêque de Saint-Malo et à MM. du chapitre, 1709, 680 p. Il est chanoine de Saint-Malo de 1662 à 1714 (Guillotin de Corson, Amédée, Pouillé historique de l'archevêché de Rennes, Rennes-Paris, Fougeray-Haton, t. I, 1880, p. 669).

111. DERIC, Gilles, Histoire ecclésiastique de la Bretagne dédiée aux seigneurs évêques de cette province, Saint-Malo, Hovius, 6 volumes, 1777-1789. Il est chanoine de Dol de 1757 (Arch. dép. d'Ille-et-Vilaine, G $164 \mathrm{~F}, \mathrm{f}^{\circ} 161 \mathrm{v}^{\circ}$ ) à la Révolution.

112. Du BreIl de PontBriant, Guillaume-Marie, Présentation aux États de l'extrait de leurs registres depuis 1732, accompagnée d'une table et projet d'une histoire des États depuis 1567 jusqu'en 1754, Rennes, Vatar, 1754, 45 p. Il est chanoine de Rennes de 1728 (Arch. dép. d'Ille-et-Vilaine, G 203 B, f ${ }^{\circ} 91$ v $^{\circ}$ ) à 1767 (Arch. diocésaines de Rennes, B 7).

113. MoreAu, J., Mémoires sur les guerres de la Ligue en Bretagne (publiées par H. WAQUET), Quimper, Archives dép. du Finistère, 1960; CHAPELAIN, J., Chronique de Lamballe, fin XVII ${ }^{\mathrm{e}}$ siècle. Le premier est chanoine de la cathédrale de Quimper, le second de la collégiale de Lamballe. 114. Armez du Poulpry, associé à la Société d'agriculture de Bretagne pour l'évêché de Saint-Brieuc en 1757, est subdélégué de l'intendant à Saint-Brieuc à partir de 1769. KERVILER, René, Répertoire général de biographie et bio-bibliographie bretonne, Rennes, Plihon et Hervé, t. 1, 1886, p. 274. 
il entre en contact avec le recteur lamballais Millet - qu'il a fréquenté au sein de la collégiale Saint-Guillaume -, avec Corgne, Le Brigant, La Tourd'Auvergne, Ogée, quelques parlementaires rennais ${ }^{115}$ et, vraisemblablement, Bagot ${ }^{116}$. À un troisième cercle appartiennent des contacts éloignés et ponctuels comme Abeille, La Chesnaye ainsi que divers correspondants comme Servière de Hesdin dans le nord du royaume ou Dubois des Fossés d'Arras ${ }^{117}$. Au-delà, c'est le domaine des savants bretons qu'il ne connaît vraisemblablement pas mais dont il fréquente les travaux : Robien, Mazéas, Déric ${ }^{118} \ldots$

Dernière remarque, et non des moindres, l'exemple de ChristopheMichel Ruffelet montre tout l'intérêt de la reconstitution de familles pour comprendre l'histoire sociale, culturelle et politique du monde capitulaire, et, plus largement, des élites de la province.

\footnotetext{
115. Il est à noter que Ruffelet semble apparenté au président à mortier Jean-Baptiste Le Vicomte de La Houssaye. Ce dernier est en effet l'époux d'une La Rivière du Beauchêne et une cousine de Ruffelet est mariée à un La Rivière de Beauchêne. SAULNIER, Frédéric, Le Parlement de Bretagne, 1554-1790. Répertoire alphabétique et biographique de tous les membres de la Cour, Rennes, Plihon, t. 2, 1902, notice 1194.

116. Jean-Louis Bagot (1728-1794) est chirurgien et médecin. Établi à Saint-Brieuc, il en devient le maire en 1774. Auteur des Observations médicinales et observations météorologiques effectuées à Saint-Brieuc et des Observations économiques et politiques sur le diocèse de Saint-Brieuc, il est lui aussi membre de la Société patriotique bretonne.

117. Ruffelet écrit à Abeille, inspecteur général des manufactures, à propos des publications de la Société d'agriculture; Servière est vraisemblablement un correspondant de Le Brigant - que Ruffelet connaît d'ailleurs très bien - qui souhaite s'entretenir de sciences physiques et naturelles avec le chanoine; La Chesnaye a lancé une enquête sur l'héraldique et Ruffelet a dû rencontrer l'un de ses correspondants. Du BOIS DE LA VILLERABEL, Arthur, « Le chroniqueur Ruffelet », op. cit., p. 157, 145 et 177. Dubois des Fossés avait demandé au docteur Jean-Louis Bagot de lui indiquer des gens de lettres de SaintBrieuc, Du Bors SainT-Sevrin, Dr, "Jean-Louis Bagot (1728-1794) ", Mémoires de la Société d'Émulation des Côtes-du-Nord, 1928, p. 253.

118. Il possède l'Histoire ecclésiastique de Bretagne de Déric et les Éléments de mathématique de Mazéas. Quant à Robien, président au Parlement de Bretagne de 1724 à 1756, il en connaît les travaux : dans les Annales il écrit que " ce savant, qui était associé à l'académie de Berlin, avait fait une étude particulière de l'histoire naturelle de sa patrie, et avait composé sur cet objet un ouvrage considérable, que la mort l'a empêché de donner au public. Perte irréparable pour la République des lettres et pour la province de Bretagne en particulier".
} 
Olivier CHARLES

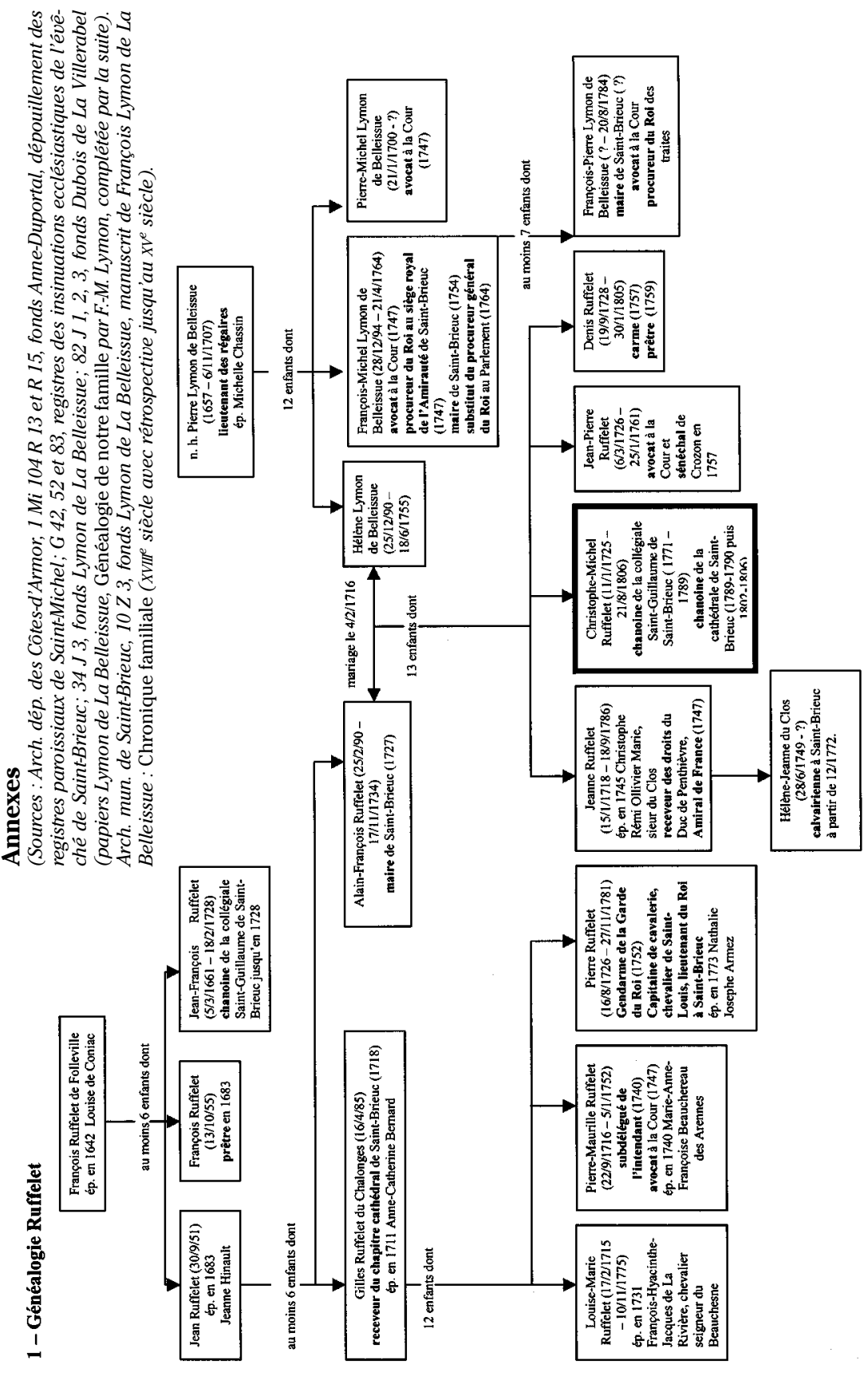




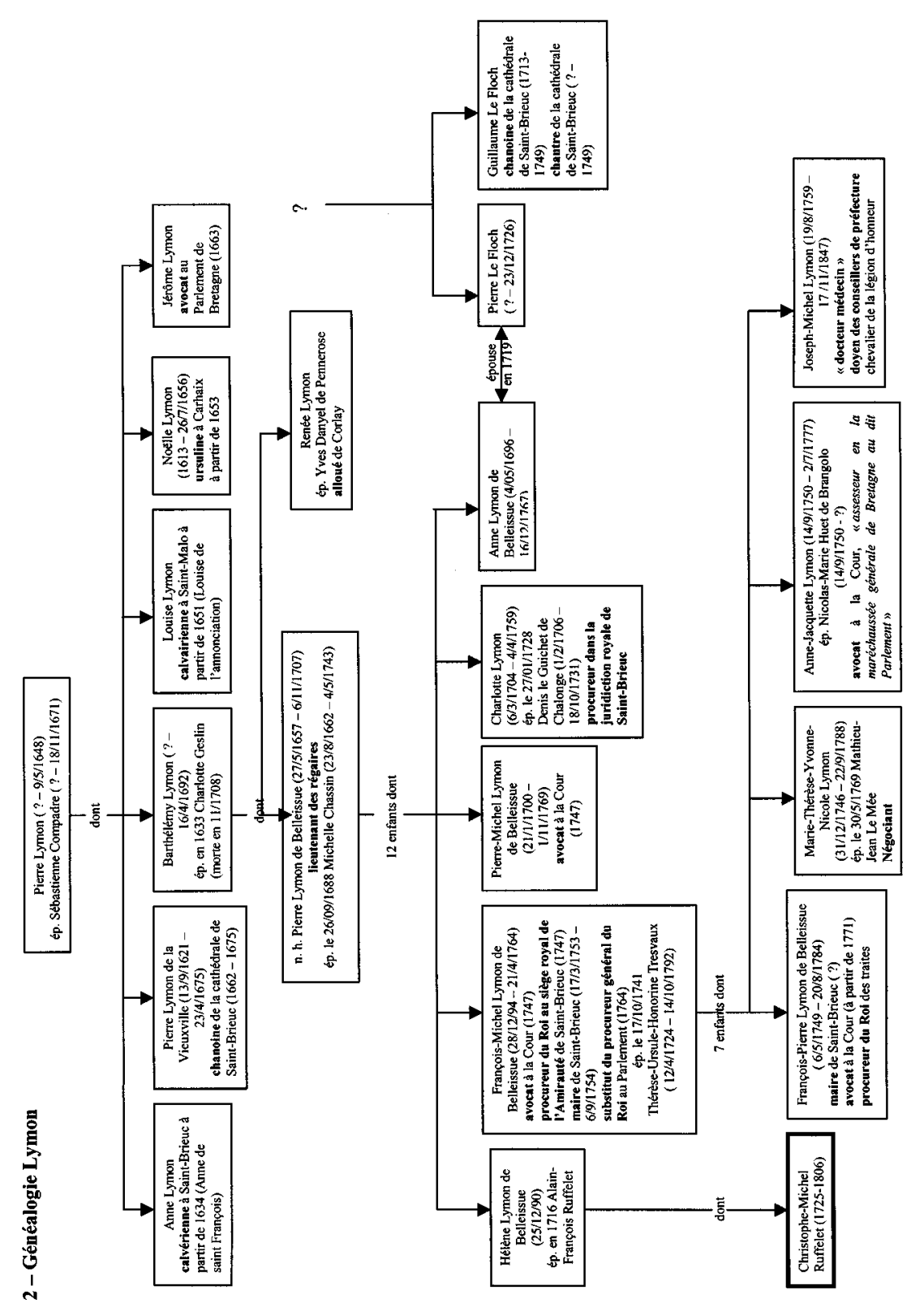




\section{RÉSUMÉ}

Les chapitres des cathédrales bretonnes d'Ancien Régime, contrairement à d'autres, n'abritent que de rares chanoines érudits. La figure du Briochin Christophe-Michel Ruffelet (1725-1806), auteur des Annales briochines, n'en est que plus précieuse et montre que l'archétype des chanoines historiens du $\mathrm{XIX}^{\mathrm{e}}$ et de la première moitié du $\mathrm{XX}^{\mathrm{e}}$ siècle peut être découvert parmi les prébendés du XVIII ${ }^{\mathrm{e}}$ siècle.

Le personnage est d'ailleurs des plus intéressants. Il est en effet le propriétaire d'une imposante bibliothèque - plus de 4000 volumes; la plus grande bibliothèque de chanoine en Bretagne - largement ouverte sur les préoccupations les plus contemporaines en histoire, en sciences, en politique... De plus, Ruffelet est un érudit qui s'intéresse autant à l'histoire la plus ancienne de la Bretagne qu'aux débats politiques les plus récents. Enfin, s'il ne se distingue pas de ses confrères par les origines, le déroulement de sa carrière - il accède tardivement au canonicat - est assez atypique.

L'étude du cas Ruffelet permet de surcroît de fortement nuancer l'image souvent dévalorisante accolée aux chanoines. Il n'est ni un dilettante, ni un ecclésiastique fermé aux évolutions de son temps, ni un conservateur impénitent entièrement tourné vers le passé. Le refus des transformations sociales et ecclésiales engendrées par la Révolution ne signifie pas refus de la modernité.

\section{ABSTRACT}

The chapters of the Breton cathedrals of the Ancien Régime, unlike others, only sheltered the odd erudite canon. The Saint-Brieuc figure, Christophe-Michel Ruffelet (1725-1806), author of the Annales briochines, is a precious example of this, and shows that the archetype of the canon historian of the $19^{\text {th }}$ and the first half of the $20^{\text {th }}$ centuries can be traced back to the prebends of the $18^{\text {th }}$ century.

He is also a character of great interest for other reasons. He owned an imposing library containing over 4000 volumes, the biggest library belonging to a canon in Brittany. He was largely aware of the concerns of his contemporaries when it came to history, sciences, politics... Moreover, Ruffelet was a scholar who showed as much interest in the most ancient history of Brittany, as he did in latest political debate. Finally, if he shared common origins with his fellow canons, the course his career took - he became a canon late in life - was far from typical.

Through studying a character like Ruffelet, we can get away from the negative image often associated with canons. He was neither a dabber, nor an ecclesiastic cut off from the changes taking place around him. Nor was he a conservative who was turned shamelessly toward the past. The refusal of social and ecclesiastical transformations brought about by the Revolution, in no way meant a refusal of modernity. 